Pacific

Journal of

Mathematics

EXISTENCE AND CONCENTRATION OF BOUND STATES OF NONLINEAR SCHRÖDINGER EQUATIONS WITH COMPACTLY SUPPORTED AND COMPETING POTENTIALS

Mingwen FeI AND Huicheng Yin

Volume $244 \quad$ No. 2

February 2010 


\title{
EXISTENCE AND CONCENTRATION OF BOUND STATES OF NONLINEAR SCHRÖDINGER EQUATIONS WITH COMPACTLY SUPPORTED AND COMPETING POTENTIALS
}

\author{
Mingwen Fei AND Huicheng Yin
}

\begin{abstract}
We study the existence and concentration of solutions to the $N$-dimensional nonlinear Schrödinger equation

$$
-\varepsilon^{2} \Delta u_{\varepsilon}+V(x) u_{\varepsilon}=K(x)\left|u_{\varepsilon}\right|^{p-1} u_{\varepsilon}+Q(x)\left|u_{\varepsilon}\right|^{q-1} u_{\varepsilon}
$$

with $u_{\varepsilon}(x)>0$ and $u_{\varepsilon} \in H^{1}\left(\mathbb{R}^{N}\right)$, where $N \geq 3,1<q<p<(N+2) /(N-2)$, and $\varepsilon>0$ is sufficiently small. We take potential functions $V(x) \in C_{0}^{\infty}\left(\mathbb{R}^{N}\right)$ with $V(x) \not \equiv$ and $V(x) \geq 0$, and show that if $K(x)$ and $Q(x)$ are permitted to be unbounded under some necessary restrictions, then a positive solution $u_{\varepsilon}(x)$ exists in $H^{1}\left(\mathbb{R}^{N}\right)$ when the corresponding ground energy function $G(x)$ has local minimum points. We establish the concentration property of $u_{\varepsilon}(x)$ as $\varepsilon$ tends to zero. We have removed from some previous papers the crucial restriction that the nonnegative potential function $V(x)$ has a positive lower bound or decays at infinity like $(1+|x|)^{-\alpha}$ with $0<\alpha \leq 2$.
\end{abstract}

\section{Introduction and statement of main results}

This paper deals with the existence and concentration of solutions to the nonlinear Schrödinger equation

(1-1) $\begin{cases}-\varepsilon^{2} \Delta u_{\varepsilon}+V(x) u_{\varepsilon}=K(x)\left|u_{\varepsilon}\right|^{p-1} u_{\varepsilon}+Q(x)\left|u_{\varepsilon}\right|^{q-1} u_{\varepsilon} & \text { for } x \in \mathbb{R}^{N}, \\ u_{\varepsilon} \in H^{1}\left(\mathbb{R}^{N}\right) & \text { for } u_{\varepsilon}(x)>0,\end{cases}$

where $N \geq 3,1<q<p<(N+2) /(N-2)$, and $\varepsilon>0$ is sufficiently small. Such solutions are called bound states in [Ambrosetti et al. 2006] and elsewhere.

Equation (1-1) has been studied extensively under various assumptions on the potential function $V(x)$ with positive lower bound and the nonlinear exponents $p$

MSC2000: primary 35J10; secondary 35J60.

Keywords: nonlinear Schrödinger equation, bound state, ground energy function, competing

potential, Harnack inequality, concentration and compactness.

This research was supported by the National Natural Science Foundation of China, numbers 10571082 and 10931007, and the National Basic Research Program of China, number 2006 CB805902.

Yin is the corresponding author. 
and $q$. See for example [Ambrosetti et al. 2003; 2004; Byeon and Wang 2003; Cao and Peng 2006; Cingolani and Lazzo 2000; del Pino and Felmer 1996; Ding and Tanaka 2003; Grossi 2002; Gui 1996; Oh 1990; Rabinowitz 1992; Wang 1993; Wang and Zeng 1997; Cingolani 2003; Floer and Weinstein 1986; Gidas et al. 1981; Kwong 1989; Lions 1984a; 1984b; Ni 1982]. In particular, due to the nonlinear terms $K(x)\left|u_{\varepsilon}\right|^{p-1} u_{\varepsilon}$ or $K(x)\left|u_{\varepsilon}\right|^{p-1} u_{\varepsilon}+Q(x)\left|u_{\varepsilon}\right|^{q-1} u_{\varepsilon}$, the concentration of $u_{\varepsilon}(x)$ can happen at some points when $\varepsilon \rightarrow 0$; in the list above, see the references listed before [Cingolani 2003]. In these works, it is usually assumed that there exists a positive constant $v_{0}$ such that

$$
V(x) \geq v_{0} \quad \text { for }|x| \gg 1 .
$$

This means that $V(x)$ has a positive lower bound at infinity.

Recently, Ambrosetti and coauthors [2005; 2007; 2006] considered a case in which $V(x)$ may decay to zero at infinity. They assumed that $V(x)$ is smooth and satisfies

$$
\frac{a}{1+|x|^{\alpha}} \leq V(x) \leq A \quad \text { in } \mathbb{R}^{N},
$$

where $a, A$ and $\alpha$ are positive constants, with $0<\alpha \leq 2$. For such situations, under $Q(x) \equiv 0$ and some restrictions on $K(x)$, they showed in [2005; 2006] that (1-1) has positive $H^{1}\left(\mathbb{R}^{N}\right)$ solutions. Furthermore, by introducing the ground energy function $G(x) \equiv V^{\theta}(x) K^{-2 /(p-1)}(x)$ with $\theta=(p+1) /(p-1)-N / 2$, they established in [2006] the concentration of $u_{\varepsilon}$ at any stable critical point of $G(x)$ and in [2005] at a global minimum point of $G(x)$ under more general hypotheses on $G(x)$.

Very recently, Yin and Zhang [2009] extended these results to the case that $V(x)$ is nonnegative but not identically zero, and established the existence of a bound state $u_{\varepsilon}$ of the equation $-\varepsilon^{2} \Delta u_{\varepsilon}+V(x) u_{\varepsilon}=K(x)\left|u_{\varepsilon}\right|^{p-1} u_{\varepsilon}$ under some sharp conditions on the unbounded nonnegative $K(x)$ in terms of different decay rates of $V(x)$ at infinity. However, they did not study the concentration property of $u_{\varepsilon}$.

This paper concerns two naturally arising questions, which are also posed in [Ambrosetti and Malchiodi 2007]: If $V(x)$ is smooth, nonnegative, and not identically zero, (that is, the assumptions (1-2) and (1-3) fail), does a bound state of (1-1) exist? And if one does, where is the concentration point of $u_{\varepsilon}(x)$ as $\varepsilon \rightarrow 0$ ? As usual, some restrictions on $K(x), Q(x)$ and $N$ are required:

$\left(H_{1}\right) V(x), K(x)$ and $Q(x)$ are smooth on $\mathbb{R}^{N}$, both $V(x)$ and $K(x)$ are nonnegative, and $V(x)$ is not identically zero.

$\left(H_{2}\right)$ There exists a smooth bounded domain $\Lambda$ of $\mathbb{R}^{N}$ on whose closure $V(x)$ and $K(x)$ are both positive, and $0<c_{0} \equiv \inf _{x \in \Lambda} G(x)<\inf _{x \in \partial \Lambda} G(x)$, where $G(x)$ is the ground energy function introduced in [Wang and Zeng 1997] 
(this will be illustrated in Section 2 below), which is positive in $\Lambda$ in the sense described in the proof of [Wang and Zeng 1997, Lemma 2.6].

$\left(H_{3}\right)$ Suppose $N \geq 5$ and $1<q<p<(N+2) /(N-2)$. Suppose also there exist positive constants $k_{1}$ and $k_{2}$ and constants $\beta_{1}<(p-1)(N-2)-2$ and $\beta_{2}<(q-1)(N-2)-2$ such that

$$
0 \leq K(x) \leq k_{1}(1+|x|)^{\beta_{1}} \quad \text { and } \quad|Q(x)| \leq k_{2}(1+|x|)^{\beta_{2}} \quad \text { in } \mathbb{R}^{N} .
$$

Theorem 1.1. For small $\varepsilon>0$, Equation (1-1) has at least one positive bound state $u_{\varepsilon}(x)$ under assumptions $\left(H_{1}\right)-\left(H_{3}\right)$,

Remark 1.2. In the general case, $\left(H_{2}\right)$ is hard to verify directly since $G(x)$ is not given explicitly, as pointed out in [Wang and Zeng 1997]. However, if $Q(x) \equiv 0$, then $\left(H_{2}\right)$ can be easily checked using the explicit formula for $G(x)$.

Remark 1.3. From $\left(H_{3}\right)$, if $p$ satisfies $(p-1)(N-2)-2>0$ and $q$ satisfies $(q-1)(N-2)-2>0$, then it is easy to see that unbounded $K(x)$ and $Q(x)$ can be permitted. On the other hand, if $1<p, q<N /(N-2)$, then $K(x)$ and $Q(x)$ should be forced to tend to zero at infinity.

Remark 1.4. The fundamental solution of the $N$-dimensional Laplacian operator is $C_{N} /|x|^{N-2}$, where $C_{N}>0$ is a suitable constant. Then in order to guarantee that $\int_{|x| \geq 1}\left(C_{N} /|x|^{N-2}\right)^{2} d x<\infty$ and that $u_{\varepsilon} \in L^{2}\left(\mathbb{R}^{N}\right)$, it is necessary to assume $N \geq 5$ in Theorem 1.1; we note that if $V(x) \approx 0$ for large $|x|$, then the properties of the linear part $-\varepsilon^{2} \Delta u_{\varepsilon}+V(x) u_{\varepsilon}$ of (1-1) are similar to those of the Laplacian $-\varepsilon^{2} \Delta u_{\varepsilon}$ for large $|x|$. On the other hand, the assumption on $\beta_{1}<(p-1)(N-2)-2$ in $\left(H_{3}\right)$ is nearly optimal for the existence of a bound state $u_{\varepsilon}(x)$ to (1-1) in the case of $Q(x) \equiv 0$, as has been shown in [Yin and Zhang 2009, Remark 1.2].

Theorem 1.5. Under assumptions $\left(H_{1}\right)-\left(H_{3}\right)$, if there exists a unique point $x_{0} \in \Lambda$ such that $G\left(x_{0}\right)=c_{0} \equiv \inf _{x \in \Lambda} G(x)$, then there exists a positive constant $C>0$ independent of $\varepsilon$ such that for any fixed $\delta>0$ and small $\varepsilon$, we have

$$
\frac{1}{C} \leq \max _{\left|x-x_{0}\right| \leq \delta} u_{\varepsilon}(x) \leq C \quad \text { and } \quad u_{\varepsilon}(x) \rightarrow 0 \text { uniformly for }\left|x-x_{0}\right| \geq \delta \text { as } \varepsilon \rightarrow 0 .
$$

Remark 1.6. Whereas Theorem 1.5 describes the concentration of $u_{\varepsilon}(x)$ when the ground energy function $G(x)$ has a unique minimum point in $\Lambda$, Theorem 5.5 describes the concentration when $G(x)$ has at least one local minimum point in $\Lambda$.

Now we comment on the proofs of Theorems 1.1 and 1.5. 
To prove Theorem 1.1, we first modify the nonlinear term of Equation (1-1) outside $\Lambda$ to

$$
\begin{aligned}
f_{\varepsilon}\left(x, u_{\varepsilon}\right)=\min \left\{K(x)\left(u_{\varepsilon}^{+}\right)^{p}+2 Q^{+}\right. & \left.(x)\left(u_{\varepsilon}^{+}\right)^{q}, \frac{\varepsilon^{3}}{1+|x|^{\theta_{0}}} u_{\varepsilon}^{+}, \frac{\varepsilon}{1+|x|^{N}}\right\} \\
& -\min \left\{|Q(x)|\left(u_{\varepsilon}^{+}\right)^{q}, \frac{\varepsilon^{3}}{1+|x|^{\theta_{0}}} u_{\varepsilon}^{+}, \frac{\varepsilon}{1+|x|^{N}}\right\},
\end{aligned}
$$

where $\theta_{0}>2$ is a constant to be chosen during the proof. We modify this term for three reasons: First, we hope that $f_{\varepsilon}\left(x, u_{\varepsilon}\right)$ coincides with the original nonlinear term for positive $u_{\varepsilon}$. Since $Q(x)$ can change sign, we arrange the terms $K(x)\left(u_{\varepsilon}^{+}\right)^{p}+2 Q^{+}(x)\left(u_{\varepsilon}^{+}\right)^{q}$ and $|Q(x)|\left(u_{\varepsilon}^{+}\right)^{q}$ in $f_{\varepsilon}\left(x, u_{\varepsilon}\right)$ so that $f_{\varepsilon}\left(x, u_{\varepsilon}\right)$ is a difference of two positive terms. Second, as in [Yin and Zhang 2009], we put the term $\varepsilon^{3} /\left(1+|x|^{\theta_{0}}\right) u_{\varepsilon}^{+}$in $f_{\varepsilon}\left(x, u_{\varepsilon}\right)$ so that the corresponding functional $I_{\varepsilon}$ of the modified equation $-\varepsilon^{2} \Delta u_{\varepsilon}+V(x) u_{\varepsilon}=g_{\varepsilon}\left(x, u_{\varepsilon}\right)$ will be well defined in the weighted Sobolev space

$$
E_{\varepsilon} \equiv\left\{u \in \mathscr{D}^{1,2}\left(\mathbb{R}^{N}\right): \int_{\mathbb{R}^{N}}\left(\varepsilon^{2}|\nabla u|^{2}+V(x)|u|^{2}\right) d x<\infty\right\}
$$

with $\mathscr{D}^{1,2}\left(\mathbb{R}^{N}\right)=\left\{u \in L^{2 N /(N-2)}\left(\mathbb{R}^{N}\right): \nabla u \in L^{2}\left(\mathbb{R}^{N}\right)\right\}$; this modification also makes $I_{\varepsilon}$ satisfy the Palais-Smale condition and preserve the mountain-pass geometry provided that $\varepsilon$ is small; see Section 2. Third, we put the term $\varepsilon /\left(1+|x|^{N}\right)$ in $f_{\varepsilon}\left(x, u_{\varepsilon}\right)$ so that the mountain-pass solution $u_{\varepsilon}$ of the modified equation can be controlled from above by a function decaying suitably outside of $\Lambda$, and so that $u_{\varepsilon}(x)$ decays as $|x| \rightarrow \infty$. From these, we can respectively conclude that

$$
K(x)\left(u_{\varepsilon}^{+}\right)^{p}+2 Q^{+}(x)\left(u_{\varepsilon}^{+}\right)^{q} \leq \frac{\varepsilon^{3}}{1+|x|^{\theta_{0}}} u_{\varepsilon}, \quad|Q(x)|\left(u_{\varepsilon}^{+}\right)^{q} \leq \frac{\varepsilon^{3}}{1+|x|^{\theta_{0}}} u_{\varepsilon}
$$

and

$$
K(x)\left(u_{\varepsilon}^{+}\right)^{p}+2 Q^{+}(x)\left(u_{\varepsilon}^{+}\right)^{q} \leq \frac{\varepsilon}{1+|x|^{N}}, \quad|Q(x)|\left(u_{\varepsilon}^{+}\right)^{q} \leq \frac{\varepsilon}{1+|x|^{N}}
$$

for $x$ outside $\Lambda$, and thus that $f_{\varepsilon}\left(x, u_{\varepsilon}\right) \equiv K(x)\left(u_{\varepsilon}^{+}\right)^{p}+Q(x)\left(u_{\varepsilon}^{+}\right)^{q}$. Such modification of the nonlinear term of nonlinear Schrödinger equations has been done before in [Ambrosetti et al. 2006; 2003; 2004; Bonheure and Van Schaftingen 2008; del Pino and Felmer 1996; Ding and Tanaka 2003; Floer and Weinstein 1986; Gui 1996; Yin and Zhang 2009]; however, these papers deal with different potentials and nonlinear terms, so their modifications differ.

Next, we derive a decay estimate for the solution $u_{\varepsilon}$ of the modified equation. To this end, as in [del Pino and Felmer 1996; Wang 1993; Wang and Zeng 1997], we will establish a concentration-compactness result and then show that the integral

$$
\varepsilon^{-N}\left(\frac{\mathbf{1}}{\mathbf{2}_{q}} \int_{\left|x-\xi_{\varepsilon}\right|>\varepsilon \rho}\left(\varepsilon^{2}\left|\nabla u_{\varepsilon}\right|^{2}+V(x) u_{\varepsilon}^{2}\right) d x+\alpha_{q}^{p} \int_{\left\{x:\left|x-\xi_{\varepsilon}\right|>\varepsilon \rho\right\} \cap \Lambda} K(x) u_{\varepsilon}^{p+1} d x\right)
$$


is small for suitable $\xi_{\varepsilon} \in \Lambda$ and some positive constant $\rho$. Here, we have introduced abbreviations for some recurring quantities:

$$
\frac{1}{2}_{p}:=\left(\frac{1}{2}-\frac{1}{p+1}\right) \quad \text { and } \quad \alpha_{q}^{p}:=\left(\frac{1}{q+1}-\frac{1}{p+1}\right) .
$$

From this integral then follows the pointwise decay property of $u_{\varepsilon}$ at infinity. In the proof, we must analyze the measure sequence $\mu_{u_{\varepsilon}}$ corresponding to some suitable scaling of $u_{\varepsilon}$, in order to show that $\mu_{u_{\varepsilon}}$ is uniformly compact with center $\xi_{\varepsilon}$, which is near some local minimum point of ground energy function $G(\xi)$ as $\varepsilon \rightarrow 0$. These results, together with some delicate estimates, complete the proof of Theorem 1.1. Some techniques in [del Pino and Felmer 1996; Wang 1993; Wang and Zeng 1997; Yin and Zhang 2009] — for instance, the truncation of the nonlinearity and the estimates of the concentration-compactness of $\mu_{u_{\varepsilon}}$ - play important roles in our paper, although our analysis is much more involved due to the compact support of $V(x)$ and the appearance of a second nonlinear term $Q(x)\left|u_{\varepsilon}\right|^{q-1} u_{\varepsilon}$ in (1-1).

To establish the concentration property of $u_{\varepsilon}$ in Theorem 1.5 , we need to analyze

$$
\varepsilon^{-N}\left(\frac{\mathbf{1}}{\mathbf{2}} q \int_{\left|x-x_{\varepsilon}\right|>\varepsilon \rho_{1}}\left(\varepsilon^{2}\left|\nabla u_{\varepsilon}\right|^{2}+V(x) u_{\varepsilon}^{2}\right) d x+\alpha_{q}^{p} \int_{\left\{x:\left|x-x_{\varepsilon}\right|>\varepsilon \rho_{1}\right\} \cap \Lambda} K(x) u_{\varepsilon}^{p+1} d x\right)
$$

for sufficiently small $\varepsilon$ and a suitable positive constant $\rho_{1}$, where $x_{\varepsilon}$ is the maximum point of $u_{\varepsilon}$ in $\mathbb{R}^{N}$. This analysis will yield a uniform positive lower bound of $u_{\varepsilon}$ near $x_{\varepsilon}$ via the weak Harnack inequality, thus completing the proof.

Our paper is organized as follows. In Section 2, we modify the nonlinear term of (1-1) outside $\Lambda$ and analyze in detail the resulting equation $-\varepsilon^{2} \Delta u_{\varepsilon}+V(x) u_{\varepsilon}=$ $g_{\varepsilon}\left(x, u_{\varepsilon}\right)$ for a suitably truncated function $g_{\varepsilon}\left(x, u_{\varepsilon}\right)$, and establish existence of $u_{\varepsilon}$ by using the mountain-pass lemma. In Section 3, we first state Proposition 3.1, which illustrates the compactness of measures related to the mountain-pass critical points of the modified equation, and use it derive an integral decay estimate inspired [Ambrosetti et al. 2005, by Lemma 17]; we further use the weak Harnack inequality to derive a pointwise decay estimate of $u_{\varepsilon}$. We then complete the proof of Theorem 1.1. In Section 4, we prove Proposition 3.1. Section 5 completes the proof of Theorem 1.5. The modified function $g_{\varepsilon}\left(x, u_{\varepsilon}\right)$ is shown to be Lipschitz in the variable $u_{\varepsilon}$ in the appendix.

Notation. $B_{r}\left(x_{0}\right)$ denotes the ball centered at $x_{0}$ with the radius $r$.

For a set $A \subset \mathbb{R}^{N}$, write $A_{\delta}=\left\{x \in \mathbb{R}^{N}: \operatorname{dist}(x, A) \leq \delta\right\}$ and $A^{\varepsilon}=\left\{\varepsilon^{-1} x: x \in A\right\}$, where $\varepsilon$ and $\delta$ are suitably small positive constants.

We denote by $C, C_{1}, \ldots$ generic positive constants depending only on $V(x)$, $K(x), Q(x), p$, and $q$.

We denote by $O(1)$ and $o(1)$ quantities that are respectively bounded and vanishing as, unless otherwise stated, $\varepsilon \rightarrow 0$. 


\section{Existence of critical points for a modified nonlinear equation}

First we recall some well-known facts. For $V(\xi), K(\xi)>0$ with $\xi \in \Lambda$, consider the system

$$
\left\{\begin{array}{l}
-\Delta u(x)+V(\xi) u(x)=K(\xi) u^{p}(x)+Q(\xi) u^{q}(x), \quad x \in \mathbb{R}^{N}, \\
u \in H^{1}\left(\mathbb{R}^{N}\right), \quad u(x)>0, \\
\lim _{|x| \rightarrow \infty} u(x)=0 .
\end{array}\right.
$$

The functional associated to (2-1) is defined as

$$
\begin{array}{rl}
I^{\xi}(u)=\frac{1}{2} \int_{\mathbb{R}^{N}}|\nabla u|^{2} & d x+\frac{1}{2} V(\xi) \int_{\mathbb{R}^{N}}|u|^{2} d x \\
& \quad-\frac{1}{p+1} K(\xi) \int_{\mathbb{R}^{N}}|u|^{p+1} d x-\frac{1}{q+1} Q(\xi) \int_{\mathbb{R}^{N}}|u|^{q+1} d x .
\end{array}
$$

In the terminology of [Wang and Zeng 1997], the function

$$
G(\xi)=\inf _{u \in M^{\xi}} I^{\xi}(u)
$$

is the ground energy function of (2-1), where $\mu^{\xi}$ is the Nehari manifold with

$$
\begin{aligned}
\mu^{\xi}=\left\{u \in H^{1}\left(\mathbb{R}^{N}\right) \backslash\{0\}: \int_{\mathbb{R}^{N}}|\nabla u|^{2} d x+V(\xi) \int_{\mathbb{R}^{N}}|u|^{2} d x\right. & \\
& \left.=K(\xi) \int_{\mathbb{R}^{N}}|u|^{p+1} d x+Q(\xi) \int_{\mathbb{R}^{N}}|u|^{q+1} d x\right\} .
\end{aligned}
$$

For more about $G(\xi)$, see [Cingolani and Lazzo 2000; Wang and Zeng 1997].

By [Gidas et al. 1981; Kwong 1989], Equation (2-1) has up to translation a unique positive $H^{1}\left(\mathbb{R}^{N}\right)$ solution $\omega(x)=\omega(V(\xi), K(\xi), Q(\xi) ; x)$, which is not only a mountain-pass critical point of the functional (2-2) but also is spherically symmetric and decays exponentially at infinity. In this case, $G(\xi)=I^{\xi}(\omega(x))$.

Let $E_{\varepsilon}$ be the class

$$
E_{\varepsilon}=\left\{u \in \mathscr{D}^{1,2}\left(\mathbb{R}^{N}\right): \int_{\mathbb{R}^{N}}\left(\varepsilon^{2}|\nabla u|^{2}+V(x)|u|^{2}\right) d x<\infty\right\}
$$

of weighted Sobolev spaces with $\mathscr{D}^{1,2}\left(\mathbb{R}^{N}\right)=\left\{u \in L^{2 N /(N-2)}\left(\mathbb{R}^{N}\right): \nabla u \in L^{2}\left(\mathbb{R}^{N}\right)\right\}$. Define the norm of $u \in E_{\varepsilon}$ by $\|u\|_{\varepsilon}=\left(\int_{\mathbb{R}^{N}}\left(\varepsilon^{2}|\nabla u|^{2}+V(x)|u|^{2}\right) d x\right)^{1 / 2}$.

Lemma 2.1. Assume that $\left(H_{1}\right)$ and $\left(H_{2}\right)$ hold for each $\varepsilon \in(0,1]$. Then there exists a positive constant $C_{1}$ independent of $\varepsilon$ such that, for $u \in E_{\varepsilon}$,

$$
\begin{aligned}
& \int_{\Lambda} K(x)|u|^{p+1} d x \leq C_{1} \varepsilon^{-N(p-1) / 2}\|u\|_{\varepsilon}^{p+1}, \\
& \int_{\Lambda}\left|Q(x)\left\|\left.u\right|^{q+1} d x \leq C_{1} \varepsilon^{-N(q-1) / 2}\right\| u \|_{\varepsilon}^{q+1} .\right.
\end{aligned}
$$


Proof. The proof uses the Sobolev embedding theorem and the positivity of $V(x)$ in $\Lambda$. Here we omit it, but see the proof of [Yin and Zhang 2009, Lemma 2.1].

To prove Theorem 1.1, we must modify (1-1) and then look for a solution to the modified equation; this method is often used in the study of the nonlinear elliptic equations. See for example [Gilbarg and Trudinger 1983, Chapter 12].

To this end, we define a function $f_{\epsilon}: \mathbb{R}^{n} \times \mathbb{R} \rightarrow \mathbb{R}$ by

$$
\begin{aligned}
f_{\varepsilon}(x, \xi)=\min \left\{K(x)\left(\xi^{+}\right)^{p}\right. & \left.+2 Q^{+}(x)\left(\xi^{+}\right)^{q}, \frac{\varepsilon^{3}}{1+|x|^{\theta_{0}}} \xi^{+}, \frac{\varepsilon}{1+|x|^{N}}\right\} \\
& -\min \left\{|Q(x)|\left(\xi^{+}\right)^{q}, \frac{\varepsilon^{3}}{1+|x|^{\theta_{0}}} \xi^{+}, \frac{\varepsilon}{1+|x|^{N}}\right\},
\end{aligned}
$$

where $\xi^{+}=\max \{\xi, 0\}$, and $\theta_{0}>2$ will be chosen later.

From Lemma A.1, we know that $f_{\varepsilon}(x, \xi)$ satisfies the global Lipschitz condition

$$
\left|f_{\varepsilon}(x, \xi)-f_{\varepsilon}(x, \eta)\right| \leq \frac{(p+q) \varepsilon^{3}}{1+|x|^{\theta_{0}}}|\xi-\eta| \quad \text { for } \xi, \eta \in \mathbb{R} .
$$

Set $g_{\varepsilon}(x, \xi)=\chi_{\Lambda}(x)\left(K(x)\left(\xi^{+}\right)^{p}+Q(x)\left(\xi^{+}\right)^{q}\right)+\left(1-\chi_{\Lambda}(x)\right) f_{\varepsilon}(x, \xi)$, where $\chi_{\Lambda}(x)$ is the characteristic function of the set $\Lambda$. By (2-7), it is easy to see that $g_{\varepsilon}(x, \xi)$ is Lipschitz continuous in the variable $\xi$.

We now consider a new nonlinear equation

$$
-\varepsilon^{2} \Delta u+V(x) u=g_{\varepsilon}(x, u) \text { for } x \in \mathbb{R}^{N},
$$

which has corresponding functional

$$
\begin{aligned}
I_{\varepsilon}(u)=\frac{1}{2}\|u\|_{\varepsilon}^{2}-\frac{1}{p+1} \int_{\Lambda} K(x) & \left(u^{+}\right)^{p+1} d x \\
& \quad-\frac{1}{q+1} \int_{\Lambda} Q(x)\left(u^{+}\right)^{q+1} d x-\int_{\mathbb{R}^{N} \backslash \Lambda} F_{\varepsilon}(x, u) d x,
\end{aligned}
$$

where $F_{\varepsilon}(x, \xi)=\left(1-\chi_{\Lambda}(x)\right) \int_{0}^{\xi} f_{\varepsilon}(x, \tau) d \tau$.

For $u \in E_{\varepsilon}$, a direct computation yields

$$
\begin{aligned}
\left|\int_{\mathbb{R}^{N} \backslash \Lambda} F_{\varepsilon}(x, u) d x\right| & \leq \int_{\mathbb{R}^{N} \backslash \Lambda} \frac{\varepsilon^{3}}{1+|x|^{\theta_{0}}} u^{2} d x \\
& \leq C \varepsilon^{3}\left(\int_{\mathbb{R}^{N} \backslash \Lambda}|u|^{2 N /(N-2)} d x\right)^{(N-2) / N} \\
& \leq C \varepsilon^{3} \int_{\mathbb{R}^{N}}|\nabla u|^{2} d x \leq C \varepsilon\|u\|_{\varepsilon}^{2},
\end{aligned}
$$

where we used that $\theta_{0}>2$. It thus follows from (2-5) and (2-9) that $I_{\varepsilon}(u)$ is well defined on $E_{\varepsilon}$, and $I_{\varepsilon} \in C^{1}\left(E_{\varepsilon}, \mathbb{R}\right)$.

Next we verify that $I_{\varepsilon}$ of (2-8) satisfies the Palais-Smale condition. 
Lemma 2.2. For small $\varepsilon>0$, if $\left\{u_{n}\right\} \subset E_{\varepsilon}$ is a sequence such that $I_{\varepsilon}\left(u_{n}\right)$ is bounded and $I_{\varepsilon}^{\prime}\left(u_{n}\right) \rightarrow 0$ as $n \rightarrow \infty$, then $\left\{u_{n}\right\}$ has a convergent subsequence.

Proof. Similar to (2-9), we have

$$
\left|\int_{\mathbb{R}^{N} \backslash \Lambda} f_{\varepsilon}(x, u) u d x\right| \leq C \varepsilon\|u\|_{\varepsilon}^{2} .
$$

Since $I_{\varepsilon}\left(u_{n}\right)$ is bounded and $I_{\varepsilon}^{\prime}\left(u_{n}\right) \rightarrow 0$, we have

$$
\begin{aligned}
& I_{\varepsilon}\left(u_{n}\right)=\frac{1}{2}\left\|u_{n}\right\|_{\varepsilon}^{2}-\frac{1}{p+1} \int_{\Lambda} K(x)\left(u_{n}^{+}\right)^{p+1} d x-\frac{1}{q+1} \int_{\Lambda} Q(x)\left(u_{n}^{+}\right)^{q+1} d x \\
& -\int_{\mathbb{R}^{N} \backslash \Lambda} F_{\varepsilon}\left(x, u_{n}\right) d x=O(1),
\end{aligned}
$$

$$
\begin{aligned}
I_{\varepsilon}^{\prime}\left(u_{n}\right) u_{n}=\left\|u_{n}\right\|_{\varepsilon}^{2}-\int_{\Lambda} K(x)\left(u_{n}^{+}\right)^{p+1} d x & -\int_{\Lambda} Q(x)\left(u_{n}^{+}\right)^{q+1} d x \\
& -\int_{\mathbb{R}^{N} \backslash \Lambda} f_{\varepsilon}\left(x, u_{n}\right) u_{n} d x=o(1)\left\|u_{n}\right\|_{\varepsilon} .
\end{aligned}
$$

Here $O(1)$ and $o(1)$ are bounded and vanishing as $n \rightarrow \infty$, respectively. Substituting (2-9) and (2-10) into (2-11) and eliminating the term $\int_{\Lambda} Q(x)\left(u_{n}^{+}\right)^{q+1} d x$ yields

$$
\mathbf{1}_{q}\left\|u_{n}\right\|_{\varepsilon}^{2}+\alpha_{q}^{p} \int_{\Lambda} K(x)\left(u_{n}^{+}\right)^{p+1} d x+O(1) \varepsilon\left\|u_{n}\right\|_{\varepsilon}^{2}=o(1)\left\|u_{n}\right\|_{\varepsilon}+O(1) .
$$

Then $(1 / 2-1 /(q+1))\left\|u_{n}\right\|_{\varepsilon}^{2}+O(1) \varepsilon\left\|u_{n}\right\|_{\varepsilon}^{2} \leq o(1)\left\|u_{n}\right\|_{\varepsilon}+O(1)$, because $p>q>1$. This leads to the boundedness of $\left\{u_{n}\right\}$ in $E_{\varepsilon}$.

Now $E_{\varepsilon} \hookrightarrow \mathscr{D}^{1,2}\left(\mathbb{R}^{N}\right) \hookrightarrow H_{\text {loc }}^{1}\left(\mathbb{R}^{N}\right)$, where $\hookrightarrow$ denotes continuous embedding, so the boundedness of $\left\{u_{n}\right\}$ in $E_{\varepsilon}$ implies that there exists $u_{0} \in E_{\varepsilon}$ satisfying, after passing to a subsequence if necessary,

$$
\begin{array}{ll}
u_{n} \rightarrow u_{0} & \text { weakly in } E_{\varepsilon}, \\
u_{n} \rightarrow u_{0} & \text { strongly in } L_{\text {loc }}^{t}\left(\mathbb{R}^{N}\right)
\end{array}
$$

for $2 \leq t<2 N /(N-2)$.

Next we show $\left\|u_{n}\right\|_{\varepsilon} \rightarrow\left\|u_{0}\right\|_{\varepsilon}$ as $n \rightarrow \infty$, which with (2-12) leads to the strong convergence of $\left\{u_{n}\right\}$ in $E_{\varepsilon}$.

By $I_{\varepsilon}^{\prime}\left(u_{n}\right) u_{0} \rightarrow 0$ and (2-12), we arrive at

$$
\begin{aligned}
o(1)=\int_{\mathbb{R}^{N}}\left(\nabla u_{n} \cdot \nabla u_{0}+\right. & \left.V(x) u_{n} u_{0}\right) d x-\int_{\Lambda} K(x)\left(u_{n}^{+}\right)^{p} u_{0} d x \\
& -\int_{\Lambda} Q(x)\left(u_{n}^{+}\right)^{q} u_{0} d x-\int_{\mathbb{R}^{N} \backslash \Lambda} f_{\varepsilon}\left(x, u_{n}\right) u_{0} d x .
\end{aligned}
$$


This implies

$$
\begin{aligned}
\left\|u_{0}\right\|_{\varepsilon}^{2}-\int_{\Lambda} K(x)\left(u_{n}^{+}\right)^{p} u_{0} d x-\int_{\Lambda} Q(x)\left(u_{n}^{+}\right)^{q} u_{0} d x & \\
& -\int_{\mathbb{R}^{N} \backslash \Lambda} f_{\varepsilon}\left(x, u_{n}\right) u_{0} d x=o(1) .
\end{aligned}
$$

In addition, from (2-11) and the boundedness of $\left\{u_{n}\right\}$, we have

$$
\begin{aligned}
\left\|u_{n}\right\|_{\varepsilon}^{2}-\int_{\Lambda} K(x)\left(u_{n}^{+}\right)^{p+1} d x-\int_{\Lambda} Q(x)\left(u_{n}^{+}\right)^{q+1} d x & \\
& -\int_{\mathbb{R}^{N} \backslash \Lambda} f_{\varepsilon}\left(x, u_{n}\right) u_{n} d x=o(1) .
\end{aligned}
$$

On the other hand, using (2-13), we find

$$
\begin{aligned}
& \lim _{n \rightarrow \infty} \int_{\Lambda} K(x)\left(u_{n}^{+}\right)^{p+1} d x=\lim _{n \rightarrow \infty} \int_{\Lambda} K(x)\left(u_{n}^{+}\right)^{p} u_{0} d x, \\
& \lim _{n \rightarrow \infty} \int_{\Lambda} Q(x)\left(u_{n}^{+}\right)^{q+1} d x=\lim _{n \rightarrow \infty} \int_{\Lambda} Q(x)\left(u_{n}^{+}\right)^{q} u_{0} d x,
\end{aligned}
$$

and for any fixed large $R>0$ (without losing generality, we assume $\Lambda \subset B_{R}(0)$ ),

$$
\lim _{n \rightarrow \infty} \int_{B_{R}(0) \backslash \Lambda} f_{\varepsilon}\left(x, u_{n}\right) u_{n} d x=\lim _{n \rightarrow \infty} \int_{B_{R}(0) \backslash \Lambda} f_{\varepsilon}\left(x, u_{n}\right) u_{0} d x .
$$

Thus, to conclude that $\left\|u_{n}\right\|_{\varepsilon} \rightarrow\left\|u_{0}\right\|_{\varepsilon}$, it follows from (2-15)-(2-18) that we need only prove that for any $\delta>0$, there exists $R>0$ such that for all $n$

$$
\left|\int_{\mathbb{R}^{N} \backslash B_{R}(0)} f_{\varepsilon}\left(x, u_{n}\right) u_{0} d x\right|<\delta \text { and }\left|\int_{\mathbb{R}^{N} \backslash B_{R}(0)} f_{\varepsilon}\left(x, u_{n}\right) u_{n} d x\right|<\delta .
$$

In fact, it suffices to check the first inequality in (2-19) since the second one is similar. As in the proof of (2-9), we have

$$
\begin{aligned}
\left|\int_{\mathbb{R}^{N} \backslash B_{R}} f_{\varepsilon}\left(x, u_{n}\right) u_{0} d x\right| & \leq \frac{C}{R^{\left(\theta_{0}-2\right) / 2}} \int_{\mathbb{R}^{N} \backslash B_{R}} \frac{\varepsilon^{3}}{1+|x|^{\theta_{0}+2 / 2}}\left|u_{n}\right|\left|u_{0}\right| d x \\
& \leq \frac{C \varepsilon}{R^{\left(\theta_{0}-2\right) / 2}}\left\|u_{n}\right\|_{\varepsilon}\left\|u_{0}\right\|_{\varepsilon} \rightarrow 0 \text { as } R \rightarrow \infty .
\end{aligned}
$$

The last estimate follows from the choice $\theta_{0}>2$ and the boundedness of $\left\{u_{n}\right\}$.

We now prove that $I_{\varepsilon}$ has the mountain-pass geometry. Let $\varepsilon>0$ be small. By (2-5) and (2-9), there is a number $r>0$ such that

$$
\begin{aligned}
I_{\varepsilon}(u) & \geq \frac{1}{2}\|u\|_{\varepsilon}^{2}-C \varepsilon^{-N(p-1) / 2}\|u\|_{\varepsilon}^{p+1}-C \varepsilon^{-N(q-1) / 2}\|u\|_{\varepsilon}^{q+1}-C \varepsilon\|u\|_{\varepsilon}^{2} \\
& \geq \frac{1}{4}\|u\|_{\varepsilon}^{2} \quad \text { for }\|u\|_{\varepsilon} \leq r .
\end{aligned}
$$


By choosing a nontrivial nonnegative smooth function $\varphi(x)$ with support in $\Lambda$, we find that

$$
I_{\varepsilon}(t \varphi)=\frac{1}{2} t^{2}\|\varphi\|_{\varepsilon}^{2}-\frac{t^{p+1}}{p+1} \int_{\Lambda} K(x) \varphi^{p+1} d x-\frac{t^{q+1}}{q+1} \int_{\Lambda} Q(x) \varphi^{q+1} d x
$$

goes to $-\infty$ as $t \rightarrow+\infty$. Therefore $I_{\varepsilon}$ has the mountain-pass geometry. Hence, by the standard theorem, we have this:

Lemma 2.3. Under the assumptions $\left(H_{1}\right)-\left(H_{3}\right)$, for small $\varepsilon>0$, the modified functional $I_{\varepsilon}$ of (2-8) has a nontrivial critical point $u_{\varepsilon} \in E_{\varepsilon}$ with level

$$
c_{\varepsilon}=\inf _{\gamma \in \Gamma_{\varepsilon}} \max _{0 \leq t \leq 1} I_{\varepsilon}(\gamma(t)),
$$

where $\Gamma_{\varepsilon}=\left\{\gamma \in C\left([0,1], E_{\varepsilon}\right): \gamma(0)=0, I_{\varepsilon}(\gamma(1))<0\right\}$.

Remark 2.4. Since $g_{\varepsilon}(x, \xi)$ is Lipschitz continuous in $\xi$ for fixed $x$, it follows from second order elliptic regularity theory that $u_{\varepsilon}$ is a strong solution of (2-8). One can also show that $u_{\varepsilon}>0$, as follows. Suppose first $I_{\varepsilon}^{\prime}\left(u_{\varepsilon}\right) u_{\varepsilon}^{-}=0$, with $u_{\varepsilon}^{-}=\max \left\{-u_{\varepsilon}, 0\right\}$. Then $\int_{\mathbb{R}^{N}}\left(\varepsilon^{2}\left|\nabla u_{\varepsilon}^{-}\right|^{2}+V(x)\left|u_{\varepsilon}^{-}\right|^{2}\right) d x=0$ and also $u_{\varepsilon}^{-}=0$. Thus, we find $u_{\varepsilon} \geq 0$. On the other hand, in Section 3 we will show that $u_{\varepsilon}$ satisfies (1-1), which can be reformulated as

$$
-\varepsilon^{2} \Delta u_{\varepsilon}+\left(V(x)+Q^{-}(x)\left|u_{\varepsilon}\right|^{q-1}\right) u_{\varepsilon}=K(x)\left|u_{\varepsilon}\right|^{p-1} u_{\varepsilon}+Q^{+}(x)\left|u_{\varepsilon}\right|^{q-1} u_{\varepsilon} \geq 0 .
$$

From this, together with $u_{\varepsilon} \geq 0$ and $u_{\varepsilon} \not \equiv 0$, we can obtain $u_{\varepsilon}(x)>0$ by using the strong maximum principle of second order elliptic equations.

In the following lemma, we obtain an upper bound on $c_{\varepsilon}$, so that we can later estimate

$$
\varepsilon^{-N} \inf _{u \in \mathcal{M}_{\varepsilon}}\left(\frac{1}{2_{p}}\|u\|_{\varepsilon}^{2}+\alpha_{q}^{p} \int_{\Lambda} K(x)\left(u^{+}\right)^{p+1} d x\right),
$$

where $M_{\varepsilon}=\left\{u \in E_{\varepsilon} \backslash\{0\}: I_{\varepsilon}^{\prime}(u) u=\|u\|_{\varepsilon}^{2}-\int_{\mathbb{R}^{N}} g_{\varepsilon}(x, u) u d x=0\right\}$. This will help prove Proposition 3.1, which will then play crucial role in obtaining the decay of $u_{\varepsilon}$ needed for the proof of Theorem 1.1.

Lemma 2.5. Under the hypotheses $\left(H_{1}\right)-\left(H_{3}\right)$, for small $\varepsilon>0$ we have

$$
c_{\varepsilon} \leq\left(c_{0}+o(1)\right) \varepsilon^{N} \quad \text { for small } \varepsilon>0,
$$

where $c_{0}$ is the constant defined in $\left(H_{2}\right)$.

Proof. For $\xi \in \Lambda$, choose $R>0$ such that $B_{R}(\xi) \subset \Lambda$. Define a smooth cutoff function $\eta: \mathbb{R}^{+} \rightarrow \mathbb{R}^{+}$such that $\eta(t)=1$ if $0 \leq t \leq R / 4$ and $\eta(t)=0$ if $t \geq R / 2$, with $\left|\eta^{\prime}(t)\right| \leq 8 / R$. Set

$$
w_{\varepsilon}(x)=\eta(|x-\xi|) \omega((x-\xi) / \varepsilon),
$$


where $\omega(x)=\omega(V(\xi), K(\xi), Q(\xi) ; x)$ is the unique positive $H^{1}\left(\mathbb{R}^{N}\right)$ solution of (2-1) that is spherically symmetric about the origin. Since $w_{\varepsilon}$ is compactly supported in $\Lambda$, we find $F_{\varepsilon}\left(x, t w_{\varepsilon}\right)=0$ for all $t \geq 0$, and there exists a $T>0$ large enough that $I_{\varepsilon}\left(T w_{\varepsilon}\right)<0$. This implies that the path $\gamma_{\varepsilon}(t)=\left\{t T w_{\varepsilon}: t \in[0,1]\right\}$ is an element of $\Gamma_{\varepsilon}$ that satisfies $c_{\varepsilon} \leq \max _{0 \leq t \leq 1} I_{\varepsilon}\left(\gamma_{\varepsilon}(t)\right)$. Recalling that $V(x), K(x)$ and $Q(x)$ are smooth functions and $\omega$ decays exponentially at infinity, we arrive at

$$
\begin{aligned}
& \int_{\mathbb{R}^{N}}\left(|\nabla(\eta(\varepsilon|y|) \omega(y))|^{2}+V(\xi+\varepsilon y)|\eta(\varepsilon|y|) \omega(y)|^{2}\right. \\
& \left.-|\nabla \omega(y)|^{2}-V(\xi) \omega^{2}(y)\right) d y=o(1), \\
& \int_{\mathbb{R}^{N}}\left(K(\xi+\varepsilon y)|\eta(\varepsilon|y|) \omega(y)|^{p+1}-K(\xi) \omega^{p+1}(y)\right) d y=o(1), \\
& \int_{\mathbb{R}^{N}}\left(Q(\xi+\varepsilon y)|\eta(\varepsilon|y|) \omega(y)|^{q+1}-Q(\xi) \omega^{q+1}(y)\right) d y=o(1) .
\end{aligned}
$$

Hence, by the change of variable $y=(x-\xi) / \varepsilon$, we have for $0 \leq t \leq 1$

$$
\begin{gathered}
I_{\varepsilon}\left(t T w_{\varepsilon}\right)=\frac{(t T)^{2}}{2} \int_{\mathbb{R}^{N}}\left(\varepsilon^{2}\left|\nabla w_{\varepsilon}\right|^{2}+V(x) w_{\varepsilon}^{2}\right) d x-\frac{(t T)^{p+1}}{p+1} \int_{\Lambda} K(x) w_{\varepsilon}^{p+1} d x \\
-\frac{(t T)^{q+1}}{q+1} \int_{\Lambda} Q(x) w_{\varepsilon}^{q+1} d x \\
=\frac{(t T)^{2}}{2} \varepsilon^{N} \int_{\mathbb{R}^{N}}\left(|\nabla(\eta(\varepsilon|y|) \omega(y))|^{2}+V(\xi+\varepsilon y)|\eta(\varepsilon|y|) \omega(y)|^{2}\right) d x \\
-\frac{(t T)^{p+1}}{p+1} \varepsilon^{N} \int_{\mathbb{R}^{N}} K(\xi+\varepsilon y)|\eta(\varepsilon|y|) \omega(y)|^{p+1} d y \\
-\frac{(t T)^{q+1}}{q+1} \varepsilon^{N} \int_{\mathbb{R}^{N}} Q(\xi+\varepsilon y)|\eta(\varepsilon|y|) \omega(y)|^{q+1} d y \\
=\varepsilon^{N}\left(\frac{(t T)^{2}}{2} \int_{\mathbb{R}^{N}}\left(|\nabla \omega|^{2}+V(\xi) \omega^{2}\right) d x-\frac{(t T)^{p+1}}{p+1} \int_{\mathbb{R}^{N}} K(\xi) \omega^{p+1} d y\right. \\
\left.-\frac{(t T)^{q+1}}{q+1} \int_{\mathbb{R}^{N}} Q(\xi) \omega^{q+1} d y+o(1)\right) .
\end{gathered}
$$

As in the argument of [Wang and Zeng 1997, Lemma 2.1], we get

$$
\begin{aligned}
\max _{0 \leq t \leq 1}\left(\frac{(t T)^{2}}{2} \int_{\mathbb{R}^{N}}\left(|\nabla \omega|^{2}+V(\xi) \omega^{2}\right) d x-\right. & \frac{(t T)^{p+1}}{p+1} \int_{\mathbb{R}^{N}} K(\xi) \omega^{p+1} d y \\
& \left.-\frac{(t T)^{q+1}}{q+1} \int_{\mathbb{R}^{N}} Q(\xi) \omega^{q+1} d y\right)=G(\xi) .
\end{aligned}
$$

So $\max _{0 \leq t \leq 1} I_{\varepsilon}\left(\gamma_{\varepsilon}(t)\right)=\max _{0 \leq t \leq 1} I_{\varepsilon}\left(t T w_{\varepsilon}\right)=\varepsilon^{N}(G(\xi)+o(1))$. Since $\xi$ is arbitrary and the smallness of $\varepsilon$ is independent of $\xi$, the proof is completed. 
For $\varepsilon>0$, the solution manifold of (2-8) is

$$
\begin{aligned}
M_{\varepsilon}=\left\{u \in E_{\varepsilon} \backslash\{0\}:\|u\|_{\varepsilon}^{2}=\int_{\Lambda} K(x)\left(u^{+}\right)^{p+1} d x\right. & +\int_{\Lambda} Q(x)\left(u^{+}\right)^{q+1} d x \\
& \left.+\int_{\mathbb{R}^{N} \backslash \Lambda} f_{\varepsilon}(x, u) u d x\right\} .
\end{aligned}
$$

Next we estimate $\varepsilon^{-N} \inf _{u \in \mathcal{M}_{\varepsilon}}\left(\frac{\mathbf{1}}{\mathbf{2}} q\|u\|_{\varepsilon}^{2}+\alpha_{q}^{p} \int_{\Lambda} K(x)\left(u^{+}\right)^{p+1} d x\right)$ as in [del Pino and Felmer 1996; Wang and Zeng 1997; Yin and Zhang 2009].

Lemma 2.6. For small $\varepsilon>0$, there exists a positive constant $c_{1}$ such that

$$
\begin{aligned}
c_{1} & \leq \varepsilon^{-N} \inf _{u \in \mathcal{M}_{\varepsilon}}\left(\frac{\mathbf{1}}{\mathbf{2}} q u u \|_{\varepsilon}^{2}+\alpha_{q}^{p} \int_{\Lambda} K(x)\left(u^{+}\right)^{p+1} d x\right) \\
& \leq \varepsilon^{-N}\left(\frac{\mathbf{1}}{\mathbf{2}_{q}}\left\|u_{\varepsilon}\right\|_{\varepsilon}^{2}+\alpha_{q}^{p} \int_{\Lambda} K(x)\left(u_{\varepsilon}^{+}\right)^{p+1} d x\right) \\
& \leq c_{0}+o(1) .
\end{aligned}
$$

Proof. By (2-5) and (2-10), for $u \in \mathcal{M}_{\varepsilon}$, we have

$$
\begin{aligned}
& \varepsilon^{-N}\|u\|_{\varepsilon}^{2}= \varepsilon^{-N} \int_{\Lambda} K(x)\left(u^{+}\right)^{p+1} d x+\varepsilon^{-N} \int_{\Lambda} Q(x)\left(u^{+}\right)^{q+1} d x \\
& \quad+\varepsilon^{-N} \int_{\mathbb{R}^{N} \backslash \Lambda} f_{\varepsilon}(x, u) u d x \\
& \leq C \varepsilon^{-N(p+1) / 2}\|u\|_{\varepsilon}^{p+1}+C \varepsilon^{-N(q+1) / 2}\|u\|_{\varepsilon}^{q+1}+o(1) \varepsilon^{-N}\|u\|_{\varepsilon}^{2} \\
&=C\left(\varepsilon^{-N}\|u\|_{\varepsilon}^{2}\right)^{(p+1) / 2}+C\left(\varepsilon^{-N}\|u\|_{\varepsilon}^{2}\right)^{(q+1) / 2}+o(1) \varepsilon^{-N}\|u\|_{\varepsilon}^{2} .
\end{aligned}
$$

Because $p>1$ and $q>1$, this means that there exists a positive number $C$ independent of $\varepsilon$ such that $\varepsilon^{-N}\|u\|_{\varepsilon}^{2} \geq C$ for $u \in \mathcal{M}_{\varepsilon}$. Thus we obtain the lemma's first inequality.

It follows from (2-9), (2-10) and (2-21) that

$$
\begin{aligned}
I_{\varepsilon}\left(u_{\varepsilon}\right)= & \frac{\mathbf{1}}{\mathbf{2}} q\left\|u_{\varepsilon}\right\|_{\varepsilon}^{2}+\alpha_{q}^{p} \int_{\Lambda} K(x)\left(u_{\varepsilon}^{+}\right)^{p+1} d x \\
& \quad+\frac{1}{q+1} \int_{\mathbb{R}^{N} \backslash \Lambda} f_{\varepsilon}\left(x, u_{\varepsilon}\right) u_{\varepsilon} d x-\int_{\mathbb{R}^{N} \backslash \Lambda} F_{\varepsilon}\left(x, u_{\varepsilon}\right) d x \\
= & (1+o(1))\left(\frac{1}{2}_{q}\left\|u_{\varepsilon}\right\|_{\varepsilon}^{2}+\alpha_{q}^{p} \int_{\Lambda} K(x)\left(u_{\varepsilon}^{+}\right)^{p+1} d x\right) .
\end{aligned}
$$

This together with Lemma 2.5 yields

$$
\varepsilon^{-N}\left(\frac{\mathbf{1}}{\mathbf{2}} q\left\|u_{\varepsilon}\right\|_{\varepsilon}^{2}+\alpha_{q}^{p} \int_{\Lambda} K(x)\left(u_{\varepsilon}^{+}\right)^{p+1} d x\right)=(1+o(1)) \varepsilon^{-N} I_{\varepsilon}\left(u_{\varepsilon}\right) \leq c_{0}+o(1),
$$

completing the proof. 


\section{Decay estimates and the proof of Theorem 1.1}

Let $\left\{u_{\varepsilon}\right\}$ be the solutions obtained in Lemma 2.3. In Section 4, we will prove this: Proposition 3.1. There is a sequence $\left\{\xi_{\varepsilon}\right\} \subset \Lambda$ such that for any $v>0$ there exist $\varepsilon_{1}(v), \rho_{1}(v)>0$ such that

$$
\begin{aligned}
\varepsilon^{-N}\left(\frac { \mathbf { 1 } } { \mathbf { 2 } } q \int _ { \mathbb { R } ^ { N } \backslash B _ { \varepsilon \rho _ { 1 } ( v ) } ( \xi _ { \varepsilon } ) } \left(\varepsilon^{2}\left|\nabla u_{\varepsilon}\right|^{2}\right.\right. & \left.+V(x) u_{\varepsilon}^{2}\right) d x \\
& \left.+\alpha_{q}^{p} \int_{\left(\mathbb{R}^{N} \backslash B_{\varepsilon \rho_{1}(v)}\left(\xi_{\varepsilon}\right)\right) \cap \Lambda} K(x) u_{\varepsilon}^{p+1} d x\right)<v
\end{aligned}
$$

and

$$
\operatorname{dist}\left(\xi_{\varepsilon}, M\right)<v
$$

whenever $\varepsilon<\varepsilon_{1}(v)$, where $M=\left\{\xi: G(\xi)=c_{0}\right\}$.

For later use, we introduce two fixed positive numbers $K_{0}>128$ and $c>0$ such that $c^{2} \geq 128 K_{0}^{2} /\left(d_{0}^{2} V_{1}\right)$, where $d_{0}=\operatorname{dist}(\partial \Lambda, M)>0$ and $V_{1}=\frac{1}{2} \min _{x \in \Lambda} V(x)>0$.

Set

$$
v_{0}=\min \left\{\frac{d_{0}}{K_{0}}, \frac{q-1}{2(q+1)}\left(16 C_{1}\right)^{-2 /(p-1)}, \frac{q-1}{2(q+1)}\left(16 C_{1}\right)^{-2 /(q-1)}\right\},
$$

where $C_{1}$ is defined in (2-5).

Take $\varepsilon_{2}=\min \left\{\varepsilon_{1}\left(v_{0}\right), d_{0} /\left(K_{0} \rho_{1}\left(v_{0}\right)\right),(\ln 2) / c\right\}$, where $\varepsilon_{1}\left(v_{0}\right)$ and $\rho_{1}\left(v_{0}\right)$ are the functions whose existence is ensured by Proposition 3.1. From now on, we assume $\varepsilon<\varepsilon_{2}$ and $\nu<v_{0}$ in (3-1).

It follows from (3-2) that for $\varepsilon<\varepsilon_{2}$ and $v=v_{0}$

$$
\operatorname{dist}\left(\xi_{\varepsilon}, \partial \Lambda\right)>\frac{1}{2} d_{0} \quad \text { and } \quad \varepsilon \rho_{1}\left(v_{0}\right)<d_{0} / K_{0} .
$$

Define $\Omega_{n, \varepsilon}=\mathbb{R}^{N} \backslash B_{R_{n, \varepsilon}}\left(\xi_{\varepsilon}\right)$ with $R_{n, \varepsilon}=e^{c \varepsilon n}$, and let $\tilde{n}>\hat{n}$ satisfy

$$
R_{\hat{n}-1, \varepsilon}<d_{0} / K_{0} \leq R_{\hat{n}, \varepsilon} \quad \text { and } \quad R_{\tilde{n}+2, \varepsilon} \leq d_{0} / 2<R_{\tilde{n}+3, \varepsilon} .
$$

By the second inequality of (3-3), we get $R_{n, \varepsilon} \geq R_{\hat{n}, \varepsilon} \geq d_{0} / K_{0}>\varepsilon \rho_{1}\left(v_{0}\right)$ for $n \geq \hat{n}$ and $\varepsilon<\varepsilon_{2}$, and this also yields

$$
\Omega_{n, \varepsilon} \cap B_{\varepsilon \rho_{1}\left(\nu_{0}\right)}\left(\xi_{\varepsilon}\right)=\varnothing .
$$

Let $\chi_{n, \varepsilon}(x)$ be smooth cutoff functions such that $\chi_{n, \varepsilon}(x)=0$ in $B_{R_{n, \varepsilon}}\left(\xi_{\varepsilon}\right)$ and $\chi_{n, \varepsilon}(x)=1$ in $\Omega_{n+1, \varepsilon}$, with $0 \leq \chi_{n, \varepsilon} \leq 1$ and $\left|\nabla \chi_{n, \varepsilon}\right| \leq 2 /\left(R_{n+1, \varepsilon}-R_{n, \varepsilon}\right)$.

Lemma 3.2. Under assumptions $\left(H_{1}\right),\left(H_{2}\right), \varepsilon<\varepsilon_{2}$ and $\hat{n} \leq n \leq \tilde{n}$, we have

$$
\int_{\mathbb{R}^{N}} A_{n, \varepsilon} d x \leq \frac{1}{2} \int_{\Omega_{n, \varepsilon}}\left(\varepsilon^{2}\left|\nabla u_{\varepsilon}\right|^{2}+V(x) u_{\varepsilon}^{2}\right) d x,
$$

where $A_{n, \varepsilon}(x)=\varepsilon^{2}\left|\nabla\left(\chi_{n, \varepsilon} u_{\varepsilon}\right)\right|^{2}+V(x)\left(\chi_{n, \varepsilon} u_{\varepsilon}\right)^{2}$. 
Proof. Straightforward computation gives $R_{n+1, \varepsilon}-R_{n, \varepsilon} \geq c \varepsilon R_{n+1, \varepsilon} / 2$ for $\varepsilon<\varepsilon_{2}$. This yields

$$
\varepsilon^{2}\left|\nabla \chi_{n, \varepsilon}\right|^{2} \leq \frac{4 \varepsilon^{2}}{\left|R_{n+1, \varepsilon}-R_{n, \varepsilon}\right|^{2}} \leq \frac{16}{c^{2} R_{n+1, \varepsilon}^{2}} .
$$

From the choice of $c$, for $\varepsilon<\varepsilon_{2}$ and $\hat{n} \leq n \leq \tilde{n}$, we arrive at

$$
\frac{128}{c^{2} R_{n+1, \varepsilon}^{2}} \leq \frac{128}{\frac{128 K_{0}^{2}}{d_{0}^{2} V_{1}} \cdot \frac{d_{0}^{2}}{K_{0}^{2}}}=V_{1} \leq V(x) \text { for } x \in\left\{x: R_{n, \varepsilon} \leq\left|x-\xi_{\varepsilon}\right|<R_{n+1, \varepsilon}\right\}
$$

Note that $\nabla \chi_{n, \varepsilon}$ is supported in $\left\{x: R_{n, \varepsilon} \leq\left|x-\xi_{\varepsilon}\right|<R_{n+1, \varepsilon}\right\}$. Then for $\varepsilon<\varepsilon_{2}$ and $\hat{n} \leq n \leq \tilde{n}$, we obtain from the last two inequalities that

$$
\varepsilon^{2}\left|\nabla \chi_{n, \varepsilon}\right|^{2} \leq \frac{1}{8} V(x) \quad \text { in } \mathbb{R}^{N} .
$$

Multiplying (2-8) by $\chi_{n, \varepsilon}^{2} u_{\varepsilon}$ yields $\int_{\mathbb{R}^{N}} A_{n, \varepsilon} d x=I+I I+I I I$, where

$$
\begin{aligned}
I & =\int_{\Omega_{n, \varepsilon}} \varepsilon^{2}\left|\nabla \chi_{n, \varepsilon}\right|^{2} u_{\varepsilon}^{2} d x, \\
I I & =\int_{\Lambda \cap \Omega_{n, \varepsilon}} \chi_{n, \varepsilon}^{2} K(x)\left(u_{\varepsilon}^{+}\right)^{p+1} d x+\int_{\Lambda \cap \Omega_{n, \varepsilon}} \chi_{n, \varepsilon}^{2} Q(x)\left(u_{\varepsilon}^{+}\right)^{q+1} d x, \\
I I I & =\int_{\left(\mathbb{R}^{N} \backslash \Lambda\right) \cap \Omega_{n, \varepsilon}} f_{\varepsilon}\left(x, u_{\varepsilon}\right) \chi_{n, \varepsilon}^{2} u_{\varepsilon} d x .
\end{aligned}
$$

By (3-7), we have

$$
|I| \leq \frac{1}{8} \int_{\Omega_{n, \varepsilon}} V(x) u_{\varepsilon}^{2} d x .
$$

For $|I I|$, we only need to consider the case $\Lambda \cap \Omega_{n, \varepsilon} \neq \varnothing$. In this case, there is a set $\Sigma_{n, \varepsilon}$ such that $\Lambda \subset \Sigma_{n, \varepsilon} \subset \Lambda_{r_{0}}=\left\{x: \operatorname{dist}(x, \Lambda) \leq r_{0}\right\}$, and $\Sigma_{n, \varepsilon} \cap \Omega_{n, \varepsilon}$ has the uniform cone property, where $r_{0}>0$ is a small constant such that $V(x) \geq V_{1}$ for $x \in \Lambda_{2 r_{0}}$.

By (2-5), we have

$$
\begin{aligned}
\int_{\Lambda \cap \Omega_{n, \varepsilon}} K(x) & \left(u_{\varepsilon}^{+}\right)^{p+1} d x \leq \int_{\Sigma_{n, \varepsilon} \cap \Omega_{n, \varepsilon}} K(x)\left|u_{\varepsilon}\right|^{p+1} d x \\
& \leq C_{1} \varepsilon^{-(N(p-1)) / 2}\left(\int_{\Sigma_{n, \varepsilon} \cap \Omega_{n, \varepsilon}}\left(\varepsilon^{2}\left|\nabla u_{\varepsilon}\right|^{2}+V(x) u_{\varepsilon}^{2}\right) d x\right)^{(p+1) / 2}
\end{aligned}
$$

and

$$
\begin{array}{rl}
\int_{\Lambda \cap \Omega_{n, \varepsilon}}|Q(x)|\left(u_{\varepsilon}^{+}\right)^{q+1} & d x \\
& \leq C_{1} \varepsilon^{-N(q-1) / 2}\left(\int_{\Sigma_{n, \varepsilon} \cap \Omega_{n, \varepsilon}}\left(\varepsilon^{2}\left|\nabla u_{\varepsilon}\right|^{2}+V(x) u_{\varepsilon}^{2}\right) d x\right)^{(q+1) / 2} .
\end{array}
$$


In addition, by using (3-5), we get $\Sigma_{n, \varepsilon} \cap \Omega_{n, \varepsilon} \subset \mathbb{R}^{N} \backslash B_{\varepsilon \rho_{1}\left(\nu_{0}\right)}\left(\xi_{\varepsilon}\right)$ for $\varepsilon<\varepsilon_{2}$ and $n \geq \hat{n}$. Thus, it follows from (3-1) and the definition of $\nu_{0}$ that

$$
\begin{aligned}
|I I| \leq & \left(C_{1} \varepsilon^{-N(p-1) / 2}\left(\int_{\mathbb{R}^{N} \backslash B_{\varepsilon \rho_{1}\left(v_{0}\right)}\left(\xi_{\varepsilon}\right)}\left(\varepsilon^{2}\left|\nabla u_{\varepsilon}\right|^{2}+V(x) u_{\varepsilon}^{2}\right) d x\right)^{(p-1) / 2}\right. \\
& \left.+C_{1} \varepsilon^{-N(q-1) / 2}\left(\int_{\mathbb{R}^{N} \backslash B_{\varepsilon \rho_{1}\left(v_{0}\right)}\left(\xi_{\varepsilon}\right)}\left(\varepsilon^{2}\left|\nabla u_{\varepsilon}\right|^{2}+V(x) u_{\varepsilon}^{2}\right) d x\right)^{(q-1) / 2}\right) \\
& \times \int_{\Sigma_{n, \varepsilon} \cap \Omega_{n, \varepsilon}}\left(\varepsilon^{2}\left|\nabla u_{\varepsilon}\right|^{2}+V(x) u_{\varepsilon}^{2}\right) d x \\
\leq & \frac{1}{8} \int_{\Omega_{n, \varepsilon}}\left(\varepsilon^{2}\left|\nabla u_{\varepsilon}\right|^{2}+V(x) u_{\varepsilon}^{2}\right) d x .
\end{aligned}
$$

Finally, we estimate $|I I I|$. Similar to the proof of (2-9), for $\varepsilon<\varepsilon_{2}$, we have

$$
|I I I| \leq \int_{\Omega_{n, \varepsilon}} \frac{2 \varepsilon^{3}}{1+|x|^{\theta_{0}}} u_{\varepsilon}^{2} d x \leq \frac{1}{8} \int_{\Omega_{n, \varepsilon}}\left(\varepsilon^{2}\left|\nabla u_{\varepsilon}\right|^{2}+V(x) u_{\varepsilon}^{2}\right) d x .
$$

The lemma then follow from our estimates for $I, I I$ and $I I I$.

Lemma 3.3. Under the assumptions of Lemma 3.2, for small $\varepsilon<\varepsilon_{2}$, we have

$$
\int_{\mathbb{R}^{N}}\left|\nabla\left(\chi_{\tilde{n}, \varepsilon} u_{\varepsilon}\right)\right|^{2} d x \leq C \varepsilon^{N-2} 2^{-\ln 2 /(c \varepsilon)} .
$$

Proof. By (3-6), we have

$$
\int_{\mathbb{R}^{N}} A_{n, \varepsilon} d x \leq \frac{1}{2} \int_{\Omega_{n, \varepsilon}}\left(\varepsilon^{2}\left|\nabla u_{\varepsilon}\right|^{2}+V(x) u_{\varepsilon}^{2}\right) d x \leq \frac{1}{2} \int_{\mathbb{R}^{N}} A_{n-1, \varepsilon} d x .
$$

Iterating the above process and applying (3-5), (3-6) and (3-1), we have for small $\varepsilon$

$$
\begin{aligned}
\int_{\mathbb{R}^{N}} A_{\tilde{n}, \varepsilon} d x & \leq\left(\frac{1}{2}\right)^{\tilde{n}-\hat{n}} \int_{\mathbb{R}^{N}} A_{\hat{n}, \varepsilon} d x \\
& \leq\left(\frac{1}{2}\right)^{\tilde{n}-\hat{n}+1} \int_{\Omega_{\hat{n}, \varepsilon}}\left(\varepsilon^{2}\left|\nabla u_{\varepsilon}\right|^{2}+V(x) u_{\varepsilon}^{2}\right) d x \\
& \leq\left(\frac{1}{2}\right)^{\tilde{n}-\hat{n}+1} \int_{\mathbb{R}^{N} \backslash B_{\varepsilon \rho_{1}\left(v_{0}\right)}\left(\xi_{\varepsilon}\right)}\left(\varepsilon^{2}\left|\nabla u_{\varepsilon}\right|^{2}+V(x) u_{\varepsilon}^{2}\right) d x \\
& \leq C \varepsilon^{N}\left(\frac{1}{2}\right)^{\tilde{n}-\hat{n}} \leq C \varepsilon^{N} 2^{-\ln 2 /(c \varepsilon)} .
\end{aligned}
$$

From this, we have

$$
\int_{\mathbb{R}^{N}}\left|\nabla\left(\chi_{\tilde{n}, \varepsilon} u_{\varepsilon}\right)\right|^{2} d x \leq \varepsilon^{-2} \int_{\mathbb{R}^{N}} A_{\tilde{n}, \varepsilon} d x \leq C \varepsilon^{N-2} 2^{-\ln 2 /(c \varepsilon)} .
$$

Lemma 3.4. Under the assumptions of Lemma 3.2, we have

$$
u_{\varepsilon}(x) \leq C 2^{-\ln 2 /(2 c \varepsilon)} \quad \text { for } x \in \mathbb{R}^{N} \text { such that }\left|x-\xi_{\varepsilon}\right| \geq d_{0} / 2 .
$$


Proof. By (2-8), we see $v_{\varepsilon}(x)=u_{\varepsilon}(\varepsilon x)$ is a classical solution of the equation $(3-14)-\Delta v_{\varepsilon}+V(\varepsilon x) v_{\varepsilon}=\chi_{\varepsilon}(x)\left(K(\varepsilon x) v_{\varepsilon}^{p}+Q(\varepsilon x) v_{\varepsilon}^{q}\right)+\left(1-\chi_{\varepsilon}(x)\right) f_{\varepsilon}\left(\varepsilon x, v_{\varepsilon}\right)$, where $\chi_{\varepsilon}$ is a characteristic function of $\Lambda^{\varepsilon}=\left\{\varepsilon^{-1} x: x \in \Lambda\right\}$. Let

$$
c_{\varepsilon}(x)=\chi_{\varepsilon}(x)\left(K(\varepsilon x) v_{\varepsilon}^{p-1}(x)+Q(\varepsilon x) v_{\varepsilon}^{q-1}(x)\right)+\left(1-\chi_{\varepsilon}(x)\right) \frac{2 \varepsilon^{3}}{1+|\varepsilon x|^{\theta_{0}}} .
$$

Then $v_{\varepsilon} \in H_{\mathrm{loc}}^{1}\left(\mathbb{R}^{N}\right)$ is a nonnegative weak subsolution of $\Delta v+c_{\varepsilon}(x) v=0$. Choosing $s \in(N / 2,2 N /((p-1)(N-2)))$, we see by Lemma 2.6 and $\theta_{0}>2$ that $c_{\varepsilon}(x) \in L^{s}\left(\mathbb{R}^{N}\right)$ and

$$
\begin{array}{r}
\left\|c_{\varepsilon}(x)\right\|_{L^{s}} \leq\left\|\chi_{\varepsilon}(x) K(\varepsilon x) v_{\varepsilon}^{p-1}\right\|_{L^{s}} \\
+\left\|\chi_{\varepsilon}(x) Q(\varepsilon x) v_{\varepsilon}^{q-1}\right\|_{L^{s}}+\left\|\left(1-\chi_{\varepsilon}(x)\right) \frac{2 \varepsilon^{3}}{1+|\varepsilon x|^{\theta_{0}}}\right\|_{L^{s}} \\
\leq C\left(\int_{\Lambda^{\varepsilon}}\left(\left|\nabla v_{\varepsilon}\right|^{2}+\left|v_{\varepsilon}\right|^{2}\right) d x\right)^{(p-1) / 2}+C\left(\int_{\Lambda^{\varepsilon}}\left(\left|\nabla v_{\varepsilon}\right|^{2}+\left|v_{\varepsilon}\right|^{2}\right) d x\right)^{(q-1) / 2} \\
+C \varepsilon^{3-N / s}\left(\int_{\mathbb{R}^{N} \backslash \Lambda} \frac{1}{\left(1+|y|^{\theta_{0}}\right)^{s}} d y\right)^{1 / s} \\
\leq C\left(\varepsilon^{-N} \int_{\Lambda}\left(\varepsilon^{2}\left|\nabla u_{\varepsilon}\right|^{2}+V(y)\left|u_{\varepsilon}\right|^{2}\right) d y\right)^{(p-1) / 2} \\
+C\left(\varepsilon^{-N} \int_{\Lambda}\left(\varepsilon^{2}\left|\nabla u_{\varepsilon}\right|^{2}+V(y)\left|u_{\varepsilon}\right|^{2}\right) d y\right)^{(q-1) / 2}+C,
\end{array}
$$

which is less than or equal to $C$. Here $C$ is positive and independent of $\varepsilon$, that is, the norm $\left\|c_{\varepsilon}(x)\right\|_{L^{s}}$ is uniformly bounded in $\varepsilon$. By [Gilbarg and Trudinger 1983, Theorem 8.17 and page 193], there is a constant $C$ depending only on $d_{0}$, the dimension $N$, and the $L^{s}$ bound of $c_{\varepsilon}(x)$, such that for $z \in \mathbb{R}^{N}$

$$
v_{\varepsilon}(z) \leq C\left(\int_{B_{c d_{0}}(z)} v_{\varepsilon}^{2^{*}}(y) d y\right)^{1 / 2^{*}}, \quad \text { where } 2^{*}=\frac{2 N}{N-2} .
$$

We note that $B_{\varepsilon c d_{0}}(x) \subset \Omega_{\tilde{n}+1, \varepsilon}$ for $x \in \mathbb{R}^{N}$ with $\left|x-\xi_{\varepsilon}\right| \geq d_{0} / 2$ and for small $\varepsilon$. This, together with (3-15) and Lemma 3.3, yields

$$
\begin{aligned}
u_{\varepsilon}(x) & =v_{\varepsilon}\left(\varepsilon^{-1} x\right) \leq C\left(\int_{B_{c d_{0}}\left(\varepsilon^{-1} x\right)} v_{\varepsilon}^{2^{*}}(y) d y\right)^{1 / 2^{*}} \\
& =C\left(\varepsilon^{-N} \int_{B_{\varepsilon c d_{0}}(x)} u_{\varepsilon}^{2^{*}}(z) d z\right)^{1 / 2^{*}} \\
& \leq C \varepsilon^{-(N-2) / 2}\left(\int_{\mathbb{R}^{N}}\left(\chi_{\tilde{n}, \varepsilon} u_{\varepsilon}\right)^{2^{*}}(z) d z\right)^{1 / 2^{*}} \\
& \leq C \varepsilon^{-(N-2) / 2}\left(\int_{\mathbb{R}^{N}}\left|\nabla\left(\chi_{\tilde{n}, \varepsilon} u_{\varepsilon}\right)\right|^{2}(z) d z\right)^{1 / 2} \leq C 2^{-\ln 2 /(2 c \varepsilon)} .
\end{aligned}
$$


Remark 3.5. By Lemma 3.4, for any fixed constant $\theta \geq 1$, there exists an $\varepsilon_{0}$ depending on $\theta$ such that $u_{\varepsilon}(x) \leq \varepsilon^{\theta}$ for $\left|x-\xi_{\varepsilon}\right| \geq d_{0} / 2$ whenever $\varepsilon<\varepsilon_{0}$.

Proof of Theorem 1.1. It follows from the assumption $\left(\mathrm{H}_{3}\right)$ that there exist some positive constants $\sigma_{0}, \theta_{0}, \theta_{1}, \theta_{2}$ such that

$$
\begin{aligned}
\beta_{1} & <p \sigma_{0}-N, & N-\frac{9}{4} & <\sigma_{0}<N-2, \\
2<\theta_{0} & <(p-1) \sigma_{0}-\beta_{1}, & \theta_{0} & <\left(p-\theta_{1}\right) \sigma_{0}-\beta_{1}, \\
4+2\left(p-\theta_{1}\right) & \leq\left(\theta_{1}-1\right) \theta_{2} . & \theta_{1} & >1,
\end{aligned}
$$

As in [Yin and Zhang 2009], we define the comparison function

$$
U(x)=1 /\left|x-\xi_{\varepsilon}\right|^{\sigma_{0}} \quad \text { in }\left|x-\xi_{\varepsilon}\right| \geq d_{0} / 2 .
$$

It is easy to see that $Z(x)=U(x)-\varepsilon^{2} u_{\varepsilon}(x) \geq 0$ on $\left|x-\xi_{\varepsilon}\right|=d_{0} / 2$ for small $\varepsilon$. Since $v_{\varepsilon}(x)=u_{\varepsilon}(\varepsilon x)$ vanishes at infinity by (3-15), so does $Z(x)$.

On the other hand, using the expression for $g_{\varepsilon}(x, u)$ and noting $\sigma_{0}<N-2$, we can conclude from (2-8) and Remark 3.5 for $\left|x-\xi_{\varepsilon}\right|>d_{0} / 2$ and sufficiently small $\varepsilon$ that

$$
\begin{aligned}
\Delta Z & =\Delta U-\varepsilon^{2} \Delta u_{\varepsilon} \\
& =\sigma_{0}\left(\sigma_{0}+2-N\right) \frac{1}{\left|x-\xi_{\varepsilon}\right| \sigma_{0}+2}-V(x) u_{\varepsilon}+g_{\varepsilon}\left(x, u_{\varepsilon}\right) \\
& \leq \sigma_{0}\left(\sigma_{0}+2-N\right) \frac{1}{\left|x-\xi_{\varepsilon}\right|^{\sigma_{0}+2}}+\chi_{\Lambda}(x) \varepsilon+\left(1-\chi_{\Lambda}(x)\right) \frac{2 \varepsilon}{1+|x|^{N}} \leq 0 .
\end{aligned}
$$

Thus, by the maximum principle we deduce that $u_{\varepsilon} \leq U / \varepsilon^{2}$ in $\left|x-\xi_{\varepsilon}\right|>d_{0} / 2$. This and the uniform boundedness of $\xi_{\varepsilon}$ imply

$$
u_{\varepsilon}(x) \leq \frac{1}{\varepsilon^{2}\left|x-\xi_{\varepsilon}\right|^{\sigma_{0}}} \leq \frac{C}{\varepsilon^{2}\left(1+|x|^{\sigma_{0}}\right)} \quad \text { in } \mathbb{R}^{N} \backslash \Lambda .
$$

Next we verify that $u_{\varepsilon}$ actually solves Equation (1-1).

Indeed, it follows from $\left(H_{3}\right)$, Remark 3.5 and (3-17) that for small $\varepsilon$

$$
\begin{aligned}
K(x) u_{\varepsilon}^{p} & \leq k_{1}\left(1+|x|^{\beta_{1}}\right)\left(\frac{C}{\varepsilon^{2}\left(1+|x|^{\sigma_{0}}\right)}\right)^{p-\theta_{1}} \varepsilon^{\left(\theta_{1}-1\right) \theta_{2}} u_{\varepsilon} \\
& \leq \frac{\varepsilon^{3}}{2\left(1+|x|^{\theta_{0}}\right)} u_{\varepsilon} \quad \text { in } \mathbb{R}^{N} \backslash \Lambda .
\end{aligned}
$$

Similarly, by $\left(H_{3}\right)$, Remark 3.5, (3-16), and (3-17), we obtain for small $\varepsilon$ that

$$
\begin{aligned}
& 2|Q(x)| u_{\varepsilon}^{q} \leq \frac{\varepsilon^{3}}{2\left(1+|x|^{\theta_{0}}\right)} u_{\varepsilon}, \quad K(x) u_{\varepsilon}^{p} \leq \frac{\varepsilon}{2\left(1+|x|^{N}\right)}, \\
& 2|Q(x)| u_{\varepsilon}^{q} \leq \frac{\varepsilon}{2\left(1+|x|^{N}\right)}
\end{aligned}
$$

for $x \in \mathbb{R}^{N} \backslash \Lambda$. 
Therefore $g_{\varepsilon}(x, u) \equiv K(x) u^{p}+Q(x) u^{q}$ in $\mathbb{R}^{N} \backslash \Lambda$ and $u_{\varepsilon}$ solves (1-1). Since $N-9 / 4<\sigma_{0}$, the estimate (3-17) leads to $u_{\varepsilon} \in L^{2}\left(\mathbb{R}^{N}\right)$ for $N \geq 5$.

\section{The proof of Proposition 3.1.}

Although the strategy is somewhat similar to that in [del Pino and Felmer 1996] or [Wang 1993; Wang and Zeng 1997; Yin and Zhang 2009], the appearance of the second nonlinear term $Q(x)|u|^{q-1} u$ in (1-1) and the compact support of $V(x)$ will make the analysis more involved.

Given $u \in \mathcal{M}_{\varepsilon}$ as defined in (2-21) for any domain $\Omega \subset \mathbb{R}^{N}$, we define the measure $\mu_{u}$ by

$$
\mu_{u}(\Omega)=\varepsilon^{-N}\left(\frac{\mathbf{1}}{2} \int_{\varepsilon \Omega}\left(\varepsilon^{2}|\nabla u|^{2}+V(x)|u|^{2}\right) d x+\alpha_{q}^{p} \int_{\varepsilon \Omega \cap \Lambda} K(x)\left(u^{+}\right)^{p+1} d x\right)
$$

$$
\begin{aligned}
=\frac{\mathbf{1}}{\mathbf{2}} q \int_{\Omega}\left(|\nabla u(\varepsilon x)|^{2}+V(\varepsilon x)|u(\varepsilon x)|^{2}\right) d x & \\
& \quad+\alpha_{q}^{p} \int_{\Omega \cap \varepsilon^{-1} \Lambda} K(\varepsilon x)\left(u^{+}(\varepsilon x)\right)^{p+1} d x,
\end{aligned}
$$

where $\varepsilon \Omega=\{\varepsilon x: x \in \Omega\}$ and $\varepsilon^{-1} \Lambda=\left\{\varepsilon^{-1} x: x \in \Lambda\right\}$.

By Lemma 2.6, we have $0<c_{1} \leq \inf _{u \in \mathcal{M}_{\varepsilon}} \mu_{u}\left(\mathbb{R}^{N}\right) \leq c_{0}+o(1)$. This means that there exists a subsequence $\varepsilon_{n} \rightarrow 0$ as $n \rightarrow \infty$, a sequence $u_{n} \in \mathcal{M}_{\varepsilon_{n}}$, and $b_{1} \in\left[c_{1}, c_{0}\right]$ such that

$$
\lim _{n \rightarrow \infty} \mu_{n}\left(\mathbb{R}^{N}\right)=\liminf _{\varepsilon \rightarrow 0} \inf _{u \in \mathcal{M}_{\varepsilon}} \mu_{u}\left(\mathbb{R}^{N}\right)=b_{1},
$$

where $\mu_{n}$ stands for $\mu_{u_{n}}$.

Let $v_{n}(x)=u_{n}\left(\varepsilon_{n} x\right)$. It follows from (2-10) and (4-2) that $v_{n}$ satisfies

$$
\lim _{n \rightarrow \infty}\left(\frac{1}{\mathbf{2}} p \int_{\Lambda^{n}} K\left(\varepsilon_{n} x\right)\left(v_{n}^{+}\right)^{p+1} d x+\frac{\mathbf{1}}{\mathbf{2}} q \int_{\Lambda^{n}} Q\left(\varepsilon_{n} x\right)\left(v_{n}^{+}\right)^{q+1} d x\right)=b_{1},
$$

where $\Lambda^{n}=\left\{\varepsilon_{n}^{-1} x: x \in \Lambda\right\}$.

By the concentration-compactness lemma of P. L. Lions [1984a, Lemma I.1], $\left\{\mu_{n}\right\}$ satisfies up to a subsequence one of three mutually exclusive possibilities:

(i) Vanishing: For all $\rho>0$,

$$
\lim _{n \rightarrow \infty} \sup _{\xi \in \mathbb{R}^{N}} \int_{B_{\rho}(\xi)} d \mu_{n}=0 .
$$

(ii) Dichotomy: There exist $b_{2} \in\left(0, b_{1}\right)$ such that for any $v>0$, there exist $\rho>0$, $\left\{\zeta_{n}\right\} \subset \mathbb{R}^{N}$ and $\rho_{n} \rightarrow+\infty$ with

$$
\left|\int_{B_{\rho}\left(\zeta_{n}\right)} d \mu_{n}-b_{2}\right| \leq v, \quad \int_{B_{\rho_{n}}\left(\zeta_{n}\right) \backslash B_{\rho}\left(\zeta_{n}\right)} d \mu_{n} \leq v,
$$


and

$$
\left|\int_{\mathbb{R}^{N} \backslash B_{\rho_{n}}\left(\zeta_{n}\right)} d \mu_{n}-\left(b_{1}-b_{2}\right)\right| \leq v
$$

(iii) Compactness: There exists a sequence $\left\{\zeta_{n}\right\} \subset \mathbb{R}^{N}$ such that for any $v>0$, there exists $\rho>0$ such that

$$
\int_{B_{\rho}\left(\zeta_{n}\right)} d \mu_{n} \geq b_{1}-v
$$

Lemma 4.1. For small $\varepsilon>0$, the vanishing property (i) does not occur.

Proof. First, we show that there is a positive integer $m$ independent of $\varepsilon$ such that

$$
\int_{\Lambda} K(x)\left(u^{+}\right)^{p+1} d x \leq m C_{1}\left(\frac{2(q+1)}{q-1}\right)^{(p-1) / 2} \sup _{\xi \in \Lambda}\left(\mu_{u}\left(B_{1}\left(\varepsilon^{-1} \xi\right)\right)\right)^{(p-1) / 2}\|u\|_{\varepsilon}^{2},
$$

$$
\int_{\Lambda}|Q(x)|\left(u^{+}\right)^{q+1} d x \leq m C_{1}\left(\frac{2(q+1)}{q-1}\right)^{(p-1) / 2} \sup _{\xi \in \Lambda}\left(\mu_{u}\left(B_{1}\left(\varepsilon^{-1} \xi\right)\right)\right)^{(q-1) / 2}\|u\|_{\varepsilon}^{2},
$$

for $u \in M_{\varepsilon}$, where $C_{1}$ is the constant given in Lemma 2.1, and $\varepsilon<r_{0}$, where $r_{0}>0$ is a small constant such that $V(x) \geq V_{1}$ for $x \in \Lambda_{2 r_{0}}$.

It suffices to prove the first inequality. By (2-5) and the definition of $\mu_{u}$, we have for any $\xi \in \Lambda$,

$$
\begin{aligned}
\int_{B_{\varepsilon}(\xi)} & K(x)|u|^{p+1} d x \leq C_{1} \varepsilon^{-N(p-1) / 2}\left(\int_{B_{\varepsilon}(\xi)}\left(\varepsilon^{2}|\nabla u|^{2}+V(x)|u|^{2}\right) d x\right)^{(p+1) / 2} \\
\leq & C_{1}\left(\frac{2(q+1)}{q-1}\right)^{(p-1) / 2}\left(\mu_{u}\left(B_{1}\left(\varepsilon^{-1} \xi\right)\right)\right)^{(p-1) / 2} \int_{B_{\varepsilon}(\xi)}\left(\varepsilon^{2}|\nabla u|^{2}+V(x)|u|^{2}\right) d x .
\end{aligned}
$$

Covering $\Lambda$ by a family of balls with radius $\varepsilon$ so that any point of $\Lambda$ is contained in at most $m$ balls of the family (the integer $m$ is only related to the dimension $N$ [Lions 1984a]) and summing the last inequality over this family of balls, we get

$$
\begin{aligned}
\int_{\Lambda} K(x)\left(u^{+}\right)^{p+1} d x \leq m C_{1}\left(\frac{2(q+1)}{q-1}\right)^{(p-1) / 2} & \sup _{\xi \in \Lambda}\left(\mu_{u}\left(B_{1}\left(\varepsilon^{-1} \xi\right)\right)\right)^{(p-1) / 2} \\
& \times \int_{\Lambda_{r_{0}}}\left(\varepsilon^{2}|\nabla u|^{2}+V(x)|u|^{2}\right) d x .
\end{aligned}
$$

This means that (4-8) is true. 
Then combining (2-10) with (4-8) yields for $u \in \mathcal{M}_{\varepsilon}$

$$
\begin{aligned}
& \|u\|_{\varepsilon}^{2} \leq m C_{1}\left(\frac{2(q+1)}{q-1}\right)^{(p-1) / 2} \\
& \times\left(\sup _{\xi \in \Lambda}\left(\mu_{u}\left(B_{1}\left(\varepsilon^{-1} \xi\right)\right)\right)^{(p-1) / 2}\|u\|_{\varepsilon}^{2}+\sup _{\xi \in \Lambda}\left(\mu_{u}\left(B_{1}\left(\varepsilon^{-1} \xi\right)\right)\right)^{(q-1) / 2}\|u\|_{\varepsilon}^{2}\right)+C \varepsilon\|u\|_{\varepsilon}^{2} .
\end{aligned}
$$

Note $\|u\|_{\varepsilon} \neq 0$ for $u \in \mathcal{M}_{\varepsilon}$. Then there exists a constant $C>0$ such that

$$
\sup _{\xi \in \Lambda} \mu_{u}\left(B_{1}\left(\varepsilon^{-1} \xi\right)\right) \geq C>0
$$

for $\varepsilon$ sufficiently small. In particular, $\sup _{\xi \in \Lambda} \mu_{n}\left(B_{1}\left(\varepsilon_{n}^{-1} \xi\right)\right) \geq C>0$ holds for large $n$ in (4-2). Thus, vanishing is not possible.

Lemma 4.2. For small $\varepsilon>0$, the dichotomy property (ii) does not occur.

Proof. Suppose to the contrary that the dichotomy property (ii) does occur. We now prove the following claim:

Claim. For any $v$ as in (ii), there exists an integer $N_{1}(v)$ such that

$$
\operatorname{dist}\left(\varepsilon_{n} \zeta_{n}, \Lambda\right) \leq r_{0} \quad \text { for } n>N_{1}(\nu)
$$

If (4-10) is false, then up to a subsequence, $\operatorname{dist}\left(\varepsilon_{n} \zeta_{n}, \Lambda\right) \geq r_{0}$ for all $n$.

Let $L$ be an integer satisfying $L>2\left(b_{1}-b_{2}\right)\left(3 V_{1}+8\right) /\left(V_{1} v\right)$, where here and below $V_{1}=\frac{1}{2} \min _{x \in \Lambda} V(x)$. Choose large $N_{2} \in \mathbb{N}$ such that $\varepsilon_{n}(L+\rho)<r_{0}$ for $n>N_{2}$. Then for $n>N_{2}$, we have $B_{\rho}\left(\zeta_{n}\right) \cap \Lambda_{L}^{n}=\varnothing$ and $\varepsilon_{n} \Lambda_{L}^{n} \subset \Lambda_{r_{0}}$, where we put $\Lambda_{i}^{n}=\left\{y \in \mathbb{R}^{N}: \operatorname{dist}\left(\varepsilon_{n}^{-1} \Lambda, y\right) \leq i\right\}$ for $i=1,2, \ldots, L$. Thus, by (4-5) and (4-6), we get

$$
\int_{\Lambda_{L}^{n}} d \mu_{n} \leq \int_{\mathbb{R}^{N} \backslash B_{\rho}\left(\zeta_{n}\right)} d \mu_{n} \leq \int_{B_{\rho_{n}}\left(\zeta_{n}\right) \backslash B_{\rho}\left(\zeta_{n}\right)} d \mu_{n}+\int_{\mathbb{R}^{N} \backslash B_{\rho_{n}}\left(\zeta_{n}\right)} d \mu_{n} \leq b_{1}-b_{2}+2 v \leq 2\left(b_{1}-b_{2}\right) .
$$

Thus there is an integer $l$ satisfying $1 \leq l \leq L$ such that

$$
\int_{H_{n}} d \mu_{n} \leq \frac{2\left(b_{1}-b_{2}\right)}{L}, \quad \text { where } H_{n}=\Lambda_{l}^{n} \backslash \Lambda_{l-1}^{n} \text {. }
$$

Let $\eta_{n}$ be smooth cutoff functions such that $\eta_{n}=1$ in $\Lambda_{l-1}^{n}$ and $\eta_{n}=0$ in $\mathbb{R}^{N} \backslash \Lambda_{l}^{n}$, with $0 \leq \eta_{n} \leq 1$ and $\left|\nabla \eta_{n}\right| \leq 2$. Set $\phi_{n}=\eta_{n} v_{n}$. A simple computation yields

$$
\left|\nabla \phi_{n}\right|^{2}=\left|v_{n} \nabla \eta_{n}+\eta_{n} \nabla v_{n}\right|^{2} \leq 2\left|\nabla v_{n}\right|^{2}+8\left|v_{n}\right|^{2} .
$$


Note that $\varepsilon_{n} H_{n} \subset \Lambda_{r_{0}}$ for $n>N_{2}$. Then it follows from the estimate above, (4-11), and the choice of $L$ that

$$
\begin{aligned}
\frac{1}{2} q \int_{H_{n}}\left(\left|\nabla \phi_{n}\right|^{2}+V\right. & \left.\left(\varepsilon_{n} x\right)\left|\phi_{n}\right|^{2}\right) d x \\
& \leq \mathbf{1}_{q}\left(\frac{8}{V_{1}}+3\right) \int_{H_{n}}\left(\left|\nabla v_{n}\right|^{2}+V\left(\varepsilon_{n} x\right)\left|v_{n}\right|^{2}\right) d x \\
& \leq\left(\frac{8}{V_{1}}+3\right) \frac{2\left(b_{1}-b_{2}\right)}{L} \leq v .
\end{aligned}
$$

Combining (4-6) with (4-11) and (4-12) yields

$$
\begin{array}{r}
\frac{\mathbf{1}}{\mathbf{2}} q \int_{\mathbb{R}^{N}}\left(\left|\nabla \phi_{n}\right|^{2}+V\left(\varepsilon_{n} x\right)\left|\phi_{n}\right|^{2}\right) d x+\alpha_{q}^{p} \int_{\Lambda^{n}} K\left(\varepsilon_{n} x\right)\left(\phi^{+}\right)^{p+1} d x \\
\leq b_{1}-b_{2}+3 v .
\end{array}
$$

In addition, by (2-10), (4-13) and (4-3), we have for large $n$

$$
\frac{\mathbf{1}}{2} q\left|\int_{\mathbb{R}^{N} \backslash \Lambda^{n}} f_{\varepsilon_{n}}\left(\varepsilon_{n} x, \phi_{n}\right) \phi_{n} d x\right| \leq C \varepsilon_{n}\left(b_{1}-b_{2}+3 v\right)<v,
$$

and

$$
\frac{\mathbf{1}}{\mathbf{2}}_{p} \int_{\Lambda^{n}} K\left(\varepsilon_{n} x\right)\left(\phi_{n}^{+}\right)^{p+1} d x+\frac{\mathbf{1}}{\mathbf{2}} q \int_{\Lambda^{n}} Q\left(\varepsilon_{n} x\right)\left(\phi_{n}^{+}\right)^{q+1} d x \geq b_{1}-v .
$$

It follows from $v<b_{2} / 5$ and (4-13)-(4-15) that

$$
\begin{aligned}
\int_{\mathbb{R}^{N}}\left(\left|\nabla \phi_{n}\right|^{2}+V\left(\varepsilon_{n} x\right)\left|\phi_{n}\right|^{2}\right) d x<\int_{\Lambda^{n}} K\left(\varepsilon_{n} x\right)\left(\phi_{n}^{+}\right)^{p+1} d x \\
+\int_{\Lambda^{n}} Q\left(\varepsilon_{n} x\right)\left(\phi_{n}^{+}\right)^{q+1} d x+\int_{\mathbb{R}^{N} \backslash \Lambda^{n}} f_{\varepsilon_{n}}\left(\varepsilon_{n} x, \phi_{n}\right) \phi_{n} d x .
\end{aligned}
$$

Let $\theta_{n}>0$ such that $\theta_{n} \phi_{n}\left(x / \varepsilon_{n}\right) \in M_{\varepsilon_{n}}$; Note that $\phi_{n} \not \equiv 0$ by (4-15). Then, as in [Wang and Zeng 1997], we can choose

$$
0<\theta_{n}<1 .
$$

Indeed, if we set

$$
\begin{aligned}
& F_{n}(t) \equiv I_{\varepsilon_{n}}^{\prime}\left(t \phi_{n}\left(x / \varepsilon_{n}\right)\right) t \phi_{n}\left(x / \varepsilon_{n}\right) \\
& \begin{aligned}
=t^{2}\left\|\phi_{n}\left(x / \varepsilon_{n}\right)\right\|_{\varepsilon}^{2}-t^{p+1} \int_{\Lambda} K(x)\left(\phi_{n}^{+}\left(x / \varepsilon_{n}\right)\right)^{p+1} d x \\
-t^{q+1} \int_{\Lambda} Q(x)\left(\phi_{n}^{+}\left(x / \varepsilon_{n}\right)\right)^{q+1} d x \\
\quad-\int_{\mathbb{R}^{N} \backslash \Lambda} f_{\varepsilon_{n}}\left(x, t \phi_{n}\left(x / \varepsilon_{n}\right)\right) t \phi_{n}\left(x / \varepsilon_{n}\right) d x,
\end{aligned}
\end{aligned}
$$


then it follows from $(4-16)$ that $F_{n}(1)<0$. On the other hand, it is easy see that $F_{n}(t)>0$ for $t \ll 1$. Thus, there exists $0<\theta_{n}<1$ such that $F_{n}\left(\theta_{n}\right)=0$, that is, $\theta_{n} \phi_{n}\left(x / \varepsilon_{n}\right) \in \mathcal{M}_{\varepsilon_{n}}$.

Thus, by the definition of $b_{1}$ in (4-2) and by (4-17) and (4-13), we get for large $n$

$$
\begin{aligned}
b_{1}- & 2 v \\
& \leq \frac{\mathbf{1}}{2} q \theta_{n}^{2} \int_{\mathbb{R}^{N}}\left(\left|\nabla \phi_{n}\right|^{2}+V\left(\varepsilon_{n} x\right)\left|\phi_{n}\right|^{2}\right) d x+\alpha_{q}^{p} \theta_{n}^{p+1} \int_{\Lambda^{n}} K\left(\varepsilon_{n} x\right)\left(\phi^{+}\right)^{p+1} d x \\
& <\frac{\mathbf{1}}{2} q \int_{\mathbb{R}^{N}}\left(\left|\nabla \phi_{n}\right|^{2}+V\left(\varepsilon_{n} x\right)\left|\phi_{n}\right|^{2}\right) d x+\alpha_{q}^{p} \int_{\Lambda^{n}} K\left(\varepsilon_{n} x\right)\left(\phi^{+}\right)^{p+1} d x \\
& \leq b_{1}-b_{2}+3 v .
\end{aligned}
$$

However, this contradicts that $v<b_{2} / 5$, so (4-10) is proved.

Using (4-10), we can finish the proof of Lemma 4.2. By the hypothesis of dichotomy, for each positive integer $k$ satisfying $1 / k<\min \left\{\left(b_{1}-b_{2}\right) / 2, b_{2} / 5, r_{0}\right\}$, there exist $\rho^{k}>0$, a sequence $\left\{\zeta_{n}^{k}\right\} \subset \mathbb{R}^{N}$ and a limit $\rho_{n}^{k} \rightarrow \infty$ as $n \rightarrow \infty$ such that (4-5) and (4-6) hold. Thus, it follows from (4-10) that there exists $N_{1}(k)$ such that $\operatorname{dist}\left(\varepsilon_{n} \zeta_{n}^{k}, \Lambda\right) \leq r_{0}$ for $n>N_{1}(k)$

Choose $N_{2}(k)>N_{1}(k)$ such that $\varepsilon_{N_{2}(k)}\left(\rho^{k}+1\right)<1 / k<r_{0}$ and $\rho^{k}+1<\rho_{N_{2}(k)}^{k}$. For convenience, we now write simply $\varepsilon_{N_{2}(k)}=\varepsilon_{k}$.

Set $D_{k}=D_{k, 1} \backslash D_{k, 2}$ with $D_{k, 1}=B_{\rho^{k}+1}\left(\zeta_{N_{2}(k)}^{k}\right)$ and $D_{k, 2}=B_{\rho^{k}}\left(\zeta_{N_{2}(k)}^{k}\right)$. Then we get $\varepsilon_{k} D_{k} \subset \Lambda_{2 r_{0}}$, and we conclude from (4-5) that

$$
\int_{D_{k}} d \mu_{k} \leq 1 / k
$$

Let $\eta_{k}$ be smooth cutoff functions such that $\eta_{k}=1$ in $D_{k, 2}$ and $\eta_{k}=0$ in $\mathbb{R}^{N} \backslash D_{k, 1}$, with $0 \leq \eta_{k} \leq 1$ and $\left|\nabla \eta_{k}\right| \leq 2$. Write $\phi_{k}^{1}=\eta_{k} v_{k}$ and $\phi_{k}^{2}=\left(1-\eta_{k}\right) v_{k}$, where $v_{k}=v_{N_{2}(k)}$.

Arguing as in the proof of (4-12) and taking into account (4-19), we get

$$
\begin{aligned}
\frac{\mathbf{1}}{\mathbf{2}_{q}} \int_{D_{k}}\left(\left|\nabla\left(\phi_{k}^{1}\right)\right|^{2}+V\left(\varepsilon_{k} x\right)\left|\phi_{k}^{1}\right|^{2}\right) d x+\alpha_{q}^{p} \int_{D_{k}} \cap \Lambda_{\Lambda^{k}} K\left(\varepsilon_{k} x\right)\left(\left(\phi_{k}^{1}\right)^{+}\right)^{p+1} d x \\
\leq\left(\frac{8}{V_{1}}+4\right) \int_{D_{k}} d \mu_{k} \leq \frac{1}{k}\left(\frac{8}{V_{1}}+4\right),
\end{aligned}
$$

where $\Lambda^{k}=\varepsilon_{k}^{-1} \Lambda$.

Combining this with (4-5) leads to

$$
\begin{array}{r}
\left|\frac{\mathbf{1}}{\mathbf{2}} q \int_{\mathbb{R}^{N}}\left(\left|\nabla \phi_{k}^{1}\right|^{2}+V\left(\varepsilon_{k} x\right)\left|\phi_{k}^{1}\right|^{2}\right) d x+\alpha_{q}^{p} \int_{\Lambda^{k}} K\left(\varepsilon_{k} x\right)\left(\left(\phi_{k}^{1}\right)^{+}\right)^{p+1} d x-b_{2}\right| \\
\leq \frac{1}{k}\left(\frac{8}{V_{1}}+4\right)+\frac{1}{k}=\frac{1}{k}\left(\frac{8}{V_{1}}+5\right) .
\end{array}
$$


Letting $k \rightarrow \infty$, we obtain

$$
\begin{array}{r}
\frac{\mathbf{1}}{\mathbf{2}} q \int_{\mathbb{R}^{N}}\left(\left|\nabla\left(\phi_{k}^{1}\right)\right|^{2}+V\left(\varepsilon_{k} x\right)\left|\phi_{k}^{1}\right|^{2}\right) d x+\alpha_{q}^{p} \int_{\Lambda^{k}} K\left(\varepsilon_{k} x\right)\left(\left(\phi_{k}^{1}\right)^{+}\right)^{p+1} d x \\
\rightarrow b_{2}>0
\end{array}
$$

Analogously, we have when $k \rightarrow \infty$

$$
\begin{array}{r}
\mathbf{1}_{q} \int_{\mathbb{R}^{N}}\left(\left|\nabla\left(\phi_{k}^{2}\right)\right|^{2}+V\left(\varepsilon_{k} x\right)\left|\phi_{k}^{2}\right|^{2}\right) d x+\alpha_{q}^{p} \int_{\Lambda^{k}} K\left(\varepsilon_{k} x\right)\left(\left(\phi_{k}^{2}\right)^{+}\right)^{p+1} d x \\
\rightarrow b_{1}-b_{2}>0 .
\end{array}
$$

In addition, by (2-5) and (4-19), we have

$$
\begin{aligned}
& \frac{\mathbf{1}}{\mathbf{2}} p \int_{\Lambda^{k} \cap D_{k}} K\left(\varepsilon_{k} x\right)\left(v_{k}^{+}\right)^{p+1} d x+\frac{\mathbf{1}}{\mathbf{2}} q \int_{\Lambda^{k} \cap D_{k}} Q\left(\varepsilon_{k} x\right)\left(v_{k}^{+}\right)^{q+1} d x \\
& \leq C\left(\left(\frac{1}{k}\right)^{(p+1) / 2}+\left(\frac{1}{k}\right)^{(q+1) / 2}\right) \rightarrow 0 \quad \text { as } k \rightarrow \infty .
\end{aligned}
$$

This together with (4-3) yields

(4-22) $\lim _{k \rightarrow \infty}\left(\frac{1}{2} p \int_{\Lambda^{k} \backslash D_{k}} K\left(\varepsilon_{k} x\right)\left(v_{k}^{+}\right)^{p+1} d x+\frac{1}{2} q \int_{\Lambda^{k} \backslash D_{k}} Q\left(\varepsilon_{k} x\right)\left(v_{k}^{+}\right)^{q+1} d x\right)=b_{1}$.

We note that

$$
\begin{aligned}
& \frac{\mathbf{1}}{2}_{p} \int_{\Lambda^{k} \backslash D_{k}} K\left(\varepsilon_{k} x\right)\left(v_{k}^{+}\right)^{p+1} d x+\frac{\mathbf{1}}{\mathbf{2}} q \int_{\Lambda^{k} \backslash D_{k}} Q\left(\varepsilon_{k} x\right)\left(v_{k}^{+}\right)^{q+1} d x \\
& =\frac{\mathbf{1}}{\mathbf{2}_{p}} \int_{\Lambda^{k} \cap D_{k, 2}} K\left(\varepsilon_{k} x\right)\left(\left(\phi_{k}^{1}\right)^{+}\right)^{p+1} d x+\frac{\mathbf{1}}{\mathbf{2}_{q}} \int_{\Lambda^{k} \cap D_{k, 2}} Q\left(\varepsilon_{k} x\right)\left(\left(\phi_{k}^{1}\right)^{+}\right)^{q+1} d x \\
& \quad+\frac{\mathbf{1}}{\mathbf{2}_{p}} \int_{\Lambda^{k} \cap\left(\mathbb{R}^{N} \backslash D_{k, 1}\right)} K\left(\varepsilon_{k} x\right)\left(\left(\phi_{k}^{2}\right)^{+}\right)^{p+1} d x+\frac{\mathbf{1}}{\mathbf{2}} q \int_{\Lambda^{k} \cap\left(\mathbb{R}^{N} \backslash D_{k, 1}\right)} Q\left(\varepsilon_{k} x\right)\left(\left(\phi_{k}^{2}\right)^{+}\right)^{q+1} d x .
\end{aligned}
$$

By this, by (4-3) and (4-22), and by passing to a subsequence if necessary, we see there exists a constant $b_{3}$ such that as $k \rightarrow \infty$,

$$
\frac{\mathbf{1}}{\mathbf{2}_{p}} \int_{\Lambda^{k} \cap D_{k, 2}} K\left(\varepsilon_{k} x\right)\left(\left(\phi_{k}^{1}\right)^{+}\right)^{p+1} d x+\frac{\mathbf{1}}{\mathbf{2}} q \int_{\Lambda^{k} \cap D_{k, 2}} Q\left(\varepsilon_{k} x\right)\left(\left(\phi_{k}^{1}\right)^{+}\right)^{q+1} d x \rightarrow b_{3}
$$

and

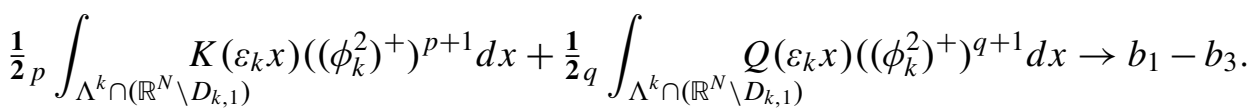

Thus, we further obtain

$$
\begin{aligned}
\frac{1}{2}_{p} \int_{\Lambda^{k}} K\left(\varepsilon_{k} x\right)\left(\left(\phi_{k}^{\lambda}\right)^{+}\right)^{p+1} d x+\frac{\mathbf{1}}{\mathbf{2}_{q}} \int_{\Lambda^{k}} Q\left(\varepsilon_{k} x\right)\left(\left(\phi_{k}^{\lambda}\right)^{+}\right)^{q+1} d x & \\
& \rightarrow \begin{cases}b_{3} & \text { if } \lambda=1, \\
b_{1}-b_{3} & \text { if } \lambda=2,\end{cases}
\end{aligned}
$$


Taking into account (2-10), (4-20), and (4-21) yields for $\lambda=1,2$

$$
\begin{aligned}
& \overline{\mathbf{1}}_{q}\left|\int_{\mathbb{R}^{N} \backslash \Lambda^{k}} f_{\varepsilon_{k}}\left(\varepsilon_{k} x, \phi_{k}^{\lambda}\right) \phi_{k}^{\lambda} d x\right| \\
& \quad=\frac{\mathbf{1}}{\mathbf{2}} \varepsilon_{k}^{-N}\left|\int_{\mathbb{R}^{N} \backslash \Lambda} f_{k}\left(y, \phi_{k}^{\lambda}\left(y / \varepsilon_{k}\right)\right) \phi_{k}^{\lambda}\left(y / \varepsilon_{k}\right) d y\right| \\
& \quad \leq C \varepsilon_{k} \times \varepsilon_{k}^{-N} \int_{\mathbb{R}^{N} \backslash \Lambda}\left(\varepsilon_{k}^{2}\left|\nabla \phi_{k}^{\lambda}\left(y / \varepsilon_{k}\right)\right|^{2}+V(y)\left|\phi_{k}^{\lambda}\left(y / \varepsilon_{k}\right)\right|^{2}\right) d y \\
& \quad=C \varepsilon_{k} \int_{\mathbb{R}^{N} \backslash \Lambda^{k}}\left(\left|\nabla \phi_{k}^{\lambda}(x)\right|^{2}+V\left(\varepsilon_{k} x\right)\left|\phi_{k}^{\lambda}(x)\right|^{2}\right) d x \rightarrow 0 \quad \text { as } k \rightarrow \infty .
\end{aligned}
$$

Therefore by (4-20), (4-21), (4-23), and (4-24), we arrive at

$$
\begin{aligned}
\int_{\mathbb{R}^{N}}\left(\left|\nabla\left(\phi_{k}^{\lambda}\right)\right|^{2}+V\left(\varepsilon_{k} x\right)\left|\phi_{k}^{\lambda}\right|^{2}\right) d x- & \int_{\Lambda^{k}} K\left(\varepsilon_{k} x\right)\left(\left(\phi_{k}^{\lambda}\right)^{+}\right)^{p+1} d x \\
-\int_{\Lambda^{k}} Q\left(\varepsilon_{k} x\right)\left(\left(\phi_{k}^{\lambda}\right)^{+}\right)^{q+1} d x- & \int_{\mathbb{R}^{N} \backslash \Lambda^{k}} f_{\varepsilon_{k}}\left(\varepsilon_{k} x, \phi_{k}^{\lambda}\right) \phi_{k}^{\lambda} d x \\
& \rightarrow \frac{2(q+1)}{q-1} \times \begin{cases}\left(b_{2}-b_{3}\right) & \text { if } \lambda=1, \\
\left(b_{3}-b_{2}\right) & \text { if } \lambda=2,\end{cases}
\end{aligned}
$$

For $\lambda=1,2$, let $\theta_{k}^{\lambda}>0$ such that $\theta_{k}^{\lambda} \phi_{k}^{\lambda}\left(x / \varepsilon_{k}\right) \in M_{\varepsilon_{k}}$. We claim that

$$
0<\theta_{k}^{\lambda} \leq 1+o(1)
$$

for at least one $\lambda$, where the quantity $o(1) \rightarrow 0$ as $k \rightarrow \infty$.

Indeed, it follows from (4-25) that if $b_{2}<b_{3}$, then for large $k$ enough

$$
\begin{aligned}
& \int_{\mathbb{R}^{N}}\left(\left|\nabla\left(\phi_{k}^{1}\right)\right|^{2}+V\left(\varepsilon_{k} x\right)\left|\phi_{k}^{1}\right|^{2}\right) d x \\
& <\int_{\Lambda^{k}} K\left(\varepsilon_{k} x\right)\left(\left(\phi_{k}^{1}\right)^{+}\right)^{p+1} d x+\int_{\Lambda^{k}} Q\left(\varepsilon_{k} x\right)\left(\left(\phi_{k}^{1}\right)^{+}\right)^{q+1} d x+\int_{\mathbb{R}^{N} \backslash \Lambda^{k}} f_{\varepsilon_{k}}\left(\varepsilon_{k} x, \phi_{k}^{1}\right) \phi_{k}^{1} d x .
\end{aligned}
$$

Analogously to the proof of (4-17), we get $0<\theta_{k}^{1}<1$. Then (4-26) holds for $\lambda=1$. If $b_{2}>b_{3}$, then by the same reasoning, we find that (4-26) holds for $\lambda=2$.

If $b_{2}=b_{3}$, as in [Wang and Zeng 1997, page 650], we will show (4-26) by way of contradiction: Without loss of generality, we assume that $\lim _{k \rightarrow \infty} \theta_{k}^{1}=\theta_{0}>1$ up to a subsequence.

Set

$$
A_{k}:=\int_{\Lambda^{k}} K\left(\varepsilon_{k} x\right)\left(\left(\phi_{k}^{1}\right)^{+}\right)^{p+1} d x \quad \text { and } \quad B_{k}:=\int_{\Lambda^{k}} Q\left(\varepsilon_{k} x\right)\left(\left(\phi_{k}^{1}\right)^{+}\right)^{q+1} d x .
$$


We now claim that up to a subsequence, $\lim _{k \rightarrow \infty}\left(A_{k}+B_{k}\right)>0$. Otherwise, it follows from (4-25) that

$$
0 \leq \lim _{k \rightarrow \infty} \int_{\mathbb{R}^{N}}\left(\left|\nabla\left(\phi_{k}^{1}\right)\right|^{2}+V\left(\varepsilon_{k} x\right)\left|\phi_{k}^{1}\right|^{2}\right) d x=\lim _{k \rightarrow \infty}\left(A_{k}+B_{k}\right) \leq 0,
$$

which implies $\lim _{k \rightarrow \infty} A_{k}=\lim _{k \rightarrow \infty} B_{k}=0$ by (2-5), contradicting (4-20). Thus $\lim _{k \rightarrow \infty}\left(A_{k}+B_{k}\right)>0$. On the other hand, by the fact $\theta_{k}^{1} \phi_{k}^{1}\left(x / \varepsilon_{k}\right) \in \mathcal{M}_{\varepsilon_{k}}$ and by (2-10), we get

$$
\lim _{k \rightarrow \infty}\left(\int_{\mathbb{R}^{N}}\left(\left|\nabla\left(\phi_{k}^{1}\right)\right|^{2}+V\left(\varepsilon_{k} x\right)\left|\phi_{k}^{1}\right|^{2}\right) d x-\theta_{k}^{p-1} A_{k}-\theta_{k}^{q-1} B_{k}\right)=0 .
$$

This and (4-25) yield

$$
\begin{aligned}
0 & =\lim _{k \rightarrow \infty}\left(\int_{\mathbb{R}^{N}}\left(\left|\nabla\left(\phi_{k}^{1}\right)\right|^{2}+V\left(\varepsilon_{k} x\right)\left|\phi_{k}^{1}\right|^{2}\right) d x-\left(A_{k}+B_{k}\right)\right) \\
& =\lim _{k \rightarrow \infty}\left(\theta_{k}^{p-1} A_{k}-\theta_{k}^{q-1} B_{k}-\left(A_{k}+B_{k}\right)\right) \\
& \geq \lim _{k \rightarrow \infty}\left(\theta_{k}^{q-1} A_{k}-\theta_{k}^{q-1} B_{k}-\left(A_{k}+B_{k}\right)\right) \\
& \geq \lim _{k \rightarrow \infty}\left(\left(\theta_{k}^{q-1}-1\right)\left(A_{k}+B_{k}\right)\right)=\left(\theta_{0}^{q-1}-1\right) \lim _{k \rightarrow \infty}\left(A_{k}+B_{k}\right)
\end{aligned}
$$

and $\theta_{0} \leq 1$, which contradict that $\theta_{0}>1$. Thus we prove (4-26).

Without loss of generality, suppose (4-26) holds for $\lambda=1$. From the definition of $b_{1}$ and (4-26), we get

$$
\begin{aligned}
b_{1}+o(1) & \leq \frac{\mathbf{1}}{\mathbf{2}_{q}}\left(\theta_{k}^{1}\right)^{2} \int_{\mathbb{R}^{N}}\left(\left|\nabla\left(\phi_{k}^{1}\right)\right|^{2}+V\left(\varepsilon_{k} x\right)\left|\phi_{k}^{1}\right|^{2}\right) d x+\alpha_{q}^{p}\left(\theta_{k}^{1}\right)^{p+1} A_{k}+o(1) \\
& \leq \frac{\mathbf{1}}{\mathbf{2}_{q}} \int_{\mathbb{R}^{N}}\left(\left|\nabla\left(\phi_{k}^{1}\right)\right|^{2}+V\left(\varepsilon_{k} x\right)\left|\phi_{k}^{1}\right|^{2}\right) d x+\alpha_{q}^{p} A_{k}+o(1) \\
& =b_{2}+o(1),
\end{aligned}
$$

which leads to a contradiction with $b_{2} \in\left(0, b_{1}\right)$. We obtain a similar contradiction in the case $\lambda=2$. Thus, the possibility of dichotomy cannot occur.

By Lemma 4.1 and Lemma 4.2, we conclude that $\left\{\mu_{n}\right\}$ is tight. That is, there exist $\left\{\zeta_{n}\right\} \subset \mathbb{R}^{N}$ such that (4-7) holds.

Lemma 4.3. We have $b_{1}=c_{0}$. In addition, up to a subsequence, $\varepsilon_{n} \zeta_{n} \rightarrow \xi_{0} \in M$.

Proof. Let $C_{1}$ be the constant in (2-5). It follows from (4-2) and (4-7) that there exists a constant $\rho_{0}>0$ and a subsequence $\left\{\zeta_{n}\right\} \subset \mathbb{R}^{N}$ such that for large $n$

$$
\int_{\mathbb{R}^{N} \backslash B_{\rho_{0}}\left(\zeta_{n}\right)} d \mu_{n} \leq \frac{\mathbf{1}}{\mathbf{2}} q_{q} \min \left\{\left(\frac{b_{1}}{4 C_{1} \frac{\mathbf{1}}{\mathbf{2}} p}\right)^{2 /(p+1)},\left(\frac{b_{1}}{4 C_{1} \frac{\mathbf{1}}{\mathbf{2}} q}\right)^{2 /(q+1)}\right\} .
$$


First we claim

$$
\operatorname{dist}\left(\varepsilon_{n} \zeta_{n}, \Lambda\right) \rightarrow 0 \quad \text { as } n \rightarrow \infty
$$

If not, there is a positive number $\delta$ such that $\operatorname{dist}\left(\varepsilon_{n} \zeta_{n}, \Lambda\right) \geq \delta$ holds up to a subsequence for all $n$. Then $B_{\rho_{0}}\left(\zeta_{n}\right) \cap \Lambda^{n}=\varnothing$ provided $n$ is large enough, where $\Lambda^{n}=\left\{\varepsilon_{n}^{-1} x: x \in \Lambda\right\}$. Then $\int_{\Lambda^{n}} d \mu_{n}$ is less than or equal to than the left side of (4-27). This fact and (2-5) yield

$$
\frac{\mathbf{1}}{\mathbf{2}_{p}} \int_{\Lambda^{n}} K\left(\varepsilon_{n} x\right)\left(v_{n}^{+}\right)^{p+1} d x+\frac{\mathbf{1}}{\mathbf{2}} q \int_{\Lambda^{n}} Q\left(\varepsilon_{n} x\right)\left(v_{n}^{+}\right)^{q+1} d x \leq \frac{1}{2} b_{1} .
$$

However, this is inconsistent with (4-3). Thus, the assertion (4-28) is true.

By (4-28), we can extract a subsequence of $\left\{\varepsilon_{n} \zeta_{n}\right\}$ (written the same for simplicity) such that

$$
\varepsilon_{n} \zeta_{n} \rightarrow \xi_{0} \in \bar{\Lambda}
$$

where $\bar{\Lambda}$ is the closure of $\Lambda$.

Set $w_{n}(x)=v_{n}^{+}\left(x+\zeta_{n}\right)$. By (4-2), we know that $\left\{w_{n}\right\}$ is bounded in $\mathscr{D}^{1,2}\left(\mathbb{R}^{N}\right)$, then, up to a subsequence, there exists $w_{0} \in \mathscr{D}^{1,2}\left(\mathbb{R}^{N}\right)$ such that

$$
\begin{array}{ll}
w_{n} \rightarrow w_{0} & \text { weakly in } \mathscr{D}^{1,2}\left(\mathbb{R}^{N}\right), \\
w_{n} \rightarrow w_{0} & \text { strongly in } L_{\text {loc }}^{p+1}\left(\mathbb{R}^{N}\right) \text { and } L_{\text {loc }}^{q+1}\left(\mathbb{R}^{N}\right), \\
w_{n} \rightarrow w_{0} & \text { almost everywhere in } \mathbb{R}^{N} .
\end{array}
$$

Applying Fatou's lemma and (4-2) yields

$$
\begin{aligned}
\int_{\mathbb{R}^{N}}\left(\left|\nabla w_{0}\right|^{2}\right. & \left.+V\left(\xi_{0}\right) w_{0}^{2}\right) d x \\
& \leq \liminf _{n \rightarrow \infty} \int_{\mathbb{R}^{N}}\left|\nabla w_{n}\right|^{2} d x+\liminf _{n \rightarrow \infty} \int_{\mathbb{R}^{N}} V\left(\varepsilon_{n} x+\varepsilon_{n} \zeta_{n}\right) w_{n}^{2} d x \\
& \leq \liminf _{n \rightarrow \infty} \int_{\mathbb{R}^{N}}\left(\left|\nabla w_{n}\right|^{2}+V\left(\varepsilon_{n} x+\varepsilon_{n} \zeta_{n}\right) w_{n}^{2}\right) d x<\infty
\end{aligned}
$$

By (4-29), we get $V\left(\xi_{0}\right)>V_{1}>0$, so it follows from (4-30) that $w_{0} \in H^{1}\left(\mathbb{R}^{N}\right)$. By the Sobolev embedding theorem, we get $w_{0}(x) \in L^{p+1}\left(\mathbb{R}^{N}\right) \cap L^{q+1}\left(\mathbb{R}^{N}\right)$. Also, given $\rho>0$, we get

$$
\begin{aligned}
& \lim _{n \rightarrow \infty} \int_{B_{\rho}(0)} K\left(\varepsilon_{n} x+\varepsilon_{n} \zeta_{n}\right) w_{n}^{p+1} d x=K\left(\xi_{0}\right) \int_{B_{\rho}(0)} w_{0}^{p+1} d x, \\
& \lim _{n \rightarrow \infty} \int_{B_{\rho}(0)} Q\left(\varepsilon_{n} x+\varepsilon_{n} \zeta_{n}\right) w_{n}^{q+1} d x=Q\left(\xi_{0}\right) \int_{B_{\rho}(0)} w_{0}^{q+1} d x .
\end{aligned}
$$

Let

$$
\Sigma_{n}:=\left\{\varepsilon_{n}^{-1} x-\zeta_{n}: x \in \Lambda\right\} \quad \text { and } \quad \Omega_{n}:=\left\{\varepsilon_{n}^{-1} x-\zeta_{n}: x \in \Lambda_{r_{0}}\right\}
$$


We have $\Sigma_{n} \subset \Omega_{n} \subset\left\{\varepsilon_{n}^{-1} x: x \in \Lambda_{2 r_{0}}\right\}$ for large $n$. For any $v>0$, the compactness of $\left\{\mu_{n}\right\}$ implies that there exists $\rho=\rho(v)>0$ such that

$$
\begin{aligned}
\int_{\Omega_{n} \backslash B_{\rho}(0)}\left(\left|\nabla w_{n}\right|^{2}+V\left(\varepsilon_{n} x+\varepsilon_{n} \zeta_{n}\right) w_{n}^{2}\right) d x & \\
\leq & \int_{\mathbb{R}^{N} \backslash B_{\rho}(0)}\left(\left|\nabla w_{n}\right|^{2}+V\left(\varepsilon_{n} x+\varepsilon_{n} \zeta_{n}\right) w_{n}^{2}\right) d x \leq \frac{2(q+1)}{q-1} v .
\end{aligned}
$$

By (4-29), there is an integer $N_{3}(v)$ with $B_{\rho}(0) \subset \Omega_{n}$ and $\operatorname{dist}\left(B_{\rho}(0), \partial \Omega_{n}\right)>1$ for $n>N_{3}(v)$; hence $\Omega_{n} \backslash B_{\rho}(0)$ has the uniform cone property. This, together with (2-5) and (4-32), yields for $n>N_{3}(v)$

$$
\begin{aligned}
& \int_{\Sigma_{n} \backslash B_{\rho}(0)} K\left(\varepsilon_{n} x+\varepsilon_{n} \zeta_{n}\right) w_{n}^{p+1}(x) d x \\
& \leq \int_{\Omega_{n} \backslash B_{\rho}(0)} K\left(\varepsilon_{n} x+\varepsilon_{n} \zeta_{n}\right) w_{n}^{p+1}(x) d x \leq C_{1}\left(\frac{2(q+1)}{q-1} v\right)^{(p+1) / 2} \\
& \int_{\Sigma_{n} \backslash B_{\rho}(0)}\left|Q\left(\varepsilon_{n} x+\varepsilon_{n} \zeta_{n}\right)\right| w_{n}^{q+1}(x) d x \leq C_{1}\left(\frac{2(q+1)}{q-1} v\right)^{(q+1) / 2} .
\end{aligned}
$$

From (4-31) and (4-33), we obtain

$$
\begin{aligned}
\lim _{n \rightarrow \infty}\left(\int_{\Sigma_{n}} K\left(\varepsilon_{n} x+\varepsilon_{n} \zeta_{n}\right) w_{n}^{p+1} d x+\int_{\Sigma_{n}} Q\left(\varepsilon_{n} x+\varepsilon_{n} \zeta_{n}\right) w_{n}^{q+1} d x\right) \\
=K\left(\xi_{0}\right) \int_{\mathbb{R}^{N}} w_{0}^{p+1} d x+Q\left(\xi_{0}\right) \int_{\mathbb{R}^{N}} w_{0}^{q+1} d x
\end{aligned}
$$

which with (4-3) implies $w_{0} \neq 0$.

Noting $u_{n} \in \mathcal{M}_{\varepsilon_{n}}$ and using (4-30), we then have

$$
\begin{aligned}
K\left(\xi_{0}\right) \int_{\mathbb{R}^{N}} w_{0}^{p+1} d x & +Q\left(\xi_{0}\right) \int_{\mathbb{R}^{N}} w_{0}^{q+1} d x \\
& \geq \liminf _{n \rightarrow \infty} \int_{\mathbb{R}^{N}}\left(\left|\nabla w_{n}\right|^{2}+V\left(\varepsilon_{n} x+\varepsilon_{n} \zeta_{n}\right) w_{n}^{2}\right) d x \\
& \geq \int_{\mathbb{R}^{N}}\left(\left|\nabla w_{0}\right|^{2}+V\left(\xi_{0}\right) w_{0}^{2}\right) d x .
\end{aligned}
$$

Now choose $\theta>0$ such that $\theta w_{0} \in \mathcal{M}^{\xi_{0}}$, where $\mathcal{M}^{\xi_{0}}$ is defined in (2-4). Then it follows from (4-35) that $\theta \leq 1$. By using the definitions of $b_{1}$ and $c_{0}$, (4-30) and (4-31), the first inequality in (4-33), and Lemma 2.6, we see that

$$
\begin{aligned}
c_{0} & \leq G\left(\xi_{0}\right) \\
& \equiv \inf _{u \in \mathcal{M}^{\xi_{0}}}\left(\frac{\mathbf{1}}{\mathbf{2}} q \int_{\mathbb{R}^{N}}\left(|\nabla u|^{2}+V\left(\xi_{0}\right) u^{2}\right) d x+\alpha_{q}^{p} K\left(\xi_{0}\right) \int_{\mathbb{R}^{N}}|u|^{p+1} d x\right)
\end{aligned}
$$




$$
\begin{aligned}
& \leq \frac{\mathbf{1}}{\mathbf{2}} q \int_{\mathbb{R}^{N}}\left(\left|\nabla\left(\theta w_{0}\right)\right|^{2}+V\left(\xi_{0}\right)\left(\theta w_{0}\right)^{2}\right) d x+\alpha_{q}^{p} K\left(\xi_{0}\right) \int_{\mathbb{R}^{N}}\left(\theta w_{0}\right)^{p+1} d x \\
& \leq \frac{\mathbf{1}}{\mathbf{2}} \int_{\mathbb{R}^{N}}\left(\left|\nabla w_{0}\right|^{2}+V\left(\xi_{0}\right) w_{0}^{2}\right) d x+\alpha_{q}^{p} K\left(\xi_{0}\right) \int_{\mathbb{R}^{N}} w_{0}^{p+1} d x \\
& \leq \liminf _{n \rightarrow \infty}\left(\frac{\mathbf{1}}{\mathbf{2}} q \int_{\mathbb{R}^{N}}\left(\left|\nabla w_{n}\right|^{2}+V\left(\varepsilon_{n} x+\varepsilon_{n} \zeta_{n}\right) w_{n}^{2}\right) d x\right. \\
& \left.\qquad+\alpha_{q}^{p} \int_{\Sigma_{n}} K\left(\varepsilon_{n} x+\varepsilon_{n} \zeta_{n}\right) w_{n}^{p+1} d x\right) \\
& \leq b_{1} \leq c_{0} .
\end{aligned}
$$

Then this yields $b_{1}=c_{0}$ and $G\left(\xi_{0}\right)=c_{0}$, which implies $\xi_{0} \in M$.

Proof of Proposition 3.1. For small $\varepsilon$, by (4-9) there exist a positive constant $C$ and $\xi_{\varepsilon} \in \Lambda$ such that

$$
\mu_{u_{\varepsilon}}\left(B_{1}\left(\varepsilon^{-1} \xi_{\varepsilon}\right)\right)>C,
$$

where $u_{\varepsilon}$ is the mountain-pass critical point of the modified (2-8), which is obtained in Lemma 2.3. We note that $\left\{\xi_{\varepsilon}\right\}$ will be chosen as the sequence in Proposition 3.1.

First we prove (3-1). If this is not true, then there exist a constant $v_{0}>0$ and limits $\varepsilon_{n} \rightarrow 0$ and $\rho_{n} \rightarrow \infty$ as $n \rightarrow \infty$ such that

$$
\int_{\mathbb{R}^{N} \backslash B_{\rho_{n}}\left(\varepsilon_{n}^{-1} \xi_{\varepsilon_{n}}\right)} d \mu_{n} \geq v_{0}>0
$$

where $\mu_{n}$ is the measure corresponding to $u_{\varepsilon_{n}}$.

By Lemma 2.6, (4-2) and Lemma 4.3, we have up to a subsequence

$$
\lim _{n \rightarrow \infty} \mu_{n}\left(\mathbb{R}^{N}\right)=c_{0} .
$$

By the arguments used to prove Lemmas 4.1 and 4.2, we conclude from (4-36) and (4-37) that $\left\{\mu_{n}\right\}$ is compact. However, as we discuss next, two exhaustive cases in P. L. Lions's concentration-compactness lemma show that $\left\{\mu_{n}\right\}$ cannot be compact.

Choose a subsequence $\left\{\zeta_{n}\right\} \subset \mathbb{R}^{N}$, and fix $\rho>0$.

Case 1. The set $B_{\rho}\left(\zeta_{n}\right) \bigcap B_{1}\left(\varepsilon_{n}^{-1} \xi_{\varepsilon_{n}}\right)$ is empty. Then $\mathbb{R}^{N} \backslash B_{\rho}\left(\zeta_{n}\right) \supset B_{1}\left(\varepsilon_{n}^{-1} \xi_{\varepsilon_{n}}\right)$, and it follows from (4-36) that $\mu_{n}\left(\mathbb{R}^{N} \backslash B_{\rho}\left(\zeta_{n}\right)\right) \geq \mu_{n}\left(B_{1}\left(\varepsilon^{-1} \xi_{\varepsilon_{n}}\right)\right)>C$.

Case 2. The set $B_{\rho}\left(\zeta_{n}\right) \bigcap B_{1}\left(\varepsilon_{n}^{-1} \xi_{\varepsilon_{n}}\right)$ is not empty. Then $\operatorname{dist}\left(\zeta_{n}, \varepsilon_{n}^{-1} \xi_{\varepsilon_{n}}\right) \leq 1+\rho$. Note that $\rho_{n} \rightarrow \infty$ as $n \rightarrow \infty$; thus $B_{\rho}\left(\zeta_{n}\right) \subset B_{\rho_{n}}\left(\varepsilon_{n}^{-1} \xi_{\varepsilon_{n}}\right)$ for large $n$. This together with (4-37) yields $\mu_{n}\left(\mathbb{R}^{N} \backslash B_{\rho}\left(\zeta_{n}\right)\right) \geq \mu_{n}\left(\mathbb{R}^{N} \backslash B_{\rho_{n}}\left(\varepsilon_{n}^{-1} \xi_{\varepsilon_{n}}\right)\right) \geq v_{0}$.

Thus, there exists a positive constant $\tilde{C}$ such that $\mu_{n}\left(\mathbb{R}^{N} \backslash B_{\rho}\left(\zeta_{n}\right)\right) \geq \tilde{C}>0$. This obviously implies $\left\{\mu_{n}\right\}$ is not compact, a contradiction that proves (3-1). 
Next we prove (3-2). If (3-2) is not true, there is a sequence $\varepsilon_{n} \rightarrow 0$ as $n \rightarrow \infty$ and a positive constant $\nu_{0}$ such that

$$
\operatorname{dist}\left(\xi_{\varepsilon_{n}}, M\right) \geq v_{0}
$$

Let $\mu_{n}$ be the measure corresponding to $u_{\varepsilon_{n}}$. By the argument above, $\left\{\mu_{n}\right\}$ is compact. Repeating the argument that proved Lemma 4.3, up to a subsequence there exists a sequence $\left\{\zeta_{n}\right\} \subset \mathbb{R}^{N}$ such that $\mu_{n}$ is concentrated in some ball centered at $\zeta_{n}$ and $\varepsilon_{n} \zeta_{n} \rightarrow \xi_{0} \in M$ as $n \rightarrow \infty$. The compactness of $\left\{\mu_{n}\right\}$ and (4-36) imply that there is a positive number $\rho_{0}$ independent of $n$ such that $\left|\zeta_{n}-\varepsilon_{n}^{-1} \xi_{\varepsilon_{n}}\right|<\rho_{0}$ (otherwise, for large $n$, we have $\mu_{n}\left(\mathbb{R}^{N} \backslash B_{\rho}\left(\zeta_{n}\right)\right) \geq \mu_{n}\left(B_{1}\left(\varepsilon_{n}^{-1} \zeta_{n}\right)\right) \geq C$, which contradicts the compactness of $\left.\left\{\mu_{n}\right\}\right)$. Hence $\left|\varepsilon_{n} \zeta_{n}-\xi_{\varepsilon_{n}}\right|<\varepsilon_{n} \rho_{0} \rightarrow 0$ as $n \rightarrow \infty$, and therefore $\xi_{\varepsilon_{n}} \rightarrow \xi_{0} \in M$. This contradicts (4-39), proving (3-2).

\section{The concentration of the bound state $u_{\varepsilon}(x)$}

We note that $u_{\varepsilon}(x)$ vanishes at infinity, so $\max _{\mathbb{R}^{N}} u_{\varepsilon}$ exists.

Lemma 5.1. For small $\varepsilon>0$, there exists a positive constant $C$ independent of $\varepsilon$ such that $\max _{\mathbb{R}^{N}} u_{\varepsilon} \geq C$.

Proof. By (2-10) and $u_{\varepsilon} \in M_{\varepsilon}$, we arrive at

$$
\begin{aligned}
\left\|u_{\varepsilon}\right\|_{\varepsilon}^{2} & =\int_{\Lambda} K(x) u_{\varepsilon}^{p+1} d x+\int_{\Lambda} Q(x) u_{\varepsilon}^{q+1} d x+\int_{\mathbb{R}^{N} \backslash \Lambda} f_{\varepsilon}\left(x, u_{\varepsilon}\right) u_{\varepsilon} d x \\
& \leq\left(\max u_{\varepsilon}\right)^{p-1} \int_{\Lambda} K(x) u_{\varepsilon}^{2} d x+\left(\max u_{\varepsilon}\right)^{q-1} \int_{\Lambda}|Q(x)| u_{\varepsilon}^{2} d x+o(1)\left\|u_{\varepsilon}\right\|_{\varepsilon}^{2} \\
& \leq C\left(\max u_{\varepsilon}\right)^{p-1}\left\|u_{\varepsilon}\right\|_{\varepsilon}^{2}+C\left(\max u_{\varepsilon}\right)^{q-1}\left\|u_{\varepsilon}\right\|_{\varepsilon}^{2}+o(1)\left\|u_{\varepsilon}\right\|_{\varepsilon}^{2} .
\end{aligned}
$$

Because $p>1$ and $q>1$, this means there is a positive number $C$ independent of $\varepsilon$ such that Lemma 5.1 holds.

Remark 5.2. Suppose $u_{\varepsilon}(x)$ obtains its maximum at the point $x=x_{\varepsilon}$, that is, $\max _{\mathbb{R}^{N}} u_{\varepsilon}(x)=u_{\varepsilon}\left(x_{\varepsilon}\right)$. Then by Remark 3.5 , we get $\left|x_{\varepsilon}-\xi_{\varepsilon}\right| \leq d_{0} / 2$ for $\varepsilon$ small enough, where $\xi_{\varepsilon}$ is given in Proposition 3.1.

Lemma 5.3. Let $x_{\varepsilon}$ be the maximum point of $u_{\varepsilon}(x)$. For any $v>0$, there exist $\varepsilon(v)>0$ and $\rho(v)>0$ such that

$$
\begin{aligned}
\varepsilon^{-N}\left(\frac { \mathbf { 1 } } { 2 } \int _ { \mathbb { R } ^ { N } \backslash B _ { \varepsilon \rho ( v ) } ( x _ { \varepsilon } ) } \left(\varepsilon^{2}\left|\nabla u_{\varepsilon}\right|^{2}\right.\right. & \left.+V(x) u_{\varepsilon}^{2}\right) d x \\
& \left.+\alpha_{q}^{p} \int_{\left(\mathbb{R}^{N} \backslash B_{\varepsilon \rho(v)}\left(x_{\varepsilon}\right)\right) \cap \Lambda} K(x) u_{\varepsilon}^{p+1} d x\right)<v
\end{aligned}
$$

and

$$
\operatorname{dist}\left(x_{\varepsilon}, M\right)<v
$$


whenever $\varepsilon<\varepsilon(v)$, where $M=\left\{\xi: C(\xi)=c_{0}\right\}$.

Proof. Firstly, we prove (5-1). Suppose it is not true. Then there exists a constant $v_{0}>0$ and limits $\varepsilon_{n} \rightarrow 0$ and $\rho_{n} \rightarrow \infty$ as $n \rightarrow \infty$ such that

$$
\int_{\mathbb{R}^{N} \backslash B_{\rho_{n}}\left(\varepsilon_{n}^{-1} x_{\varepsilon_{n}}\right)} d \mu_{n} \geq v_{0}>0
$$

where $\mu_{n}$ is the measure corresponding to $u_{\varepsilon_{n}}$, which is defined in (4-1).

We claim that

$$
\mu_{n}\left(B_{1}\left(\varepsilon_{n}^{-1} x_{\varepsilon_{n}}\right)\right) \geq C>0,
$$

where $C$ is a positive constant independent of $n$.

Let $v_{n}=u_{\varepsilon_{n}}\left(\varepsilon_{n} x\right)$. Then for large $n(5-4)$ is equivalent to (5-5) $\frac{\mathbf{1}}{\mathbf{2}} q \int_{B_{1}\left(\varepsilon_{n}^{-1} x_{\varepsilon_{n}}\right)}\left(\left|\nabla v_{n}\right|^{2}+V\left(\varepsilon_{n} x\right) v_{n}^{2}\right) d x+\alpha_{q}^{p} \int_{B_{1}\left(\varepsilon_{n}^{-1} x_{\varepsilon_{n}}\right)} K\left(\varepsilon_{n} x\right) v_{n}^{p+1} d x \geq C$.

By $q<p$ and the nonnegativity of $K(x)$, we may prove (5-4) and (5-5) by showing that

$$
\int_{B_{1}\left(\varepsilon_{n}^{-1} x_{\varepsilon_{n}}\right)}\left(\left|\nabla v_{n}\right|^{2}+v_{n}^{2}\right) d x \geq C .
$$

Since $v_{n} \geq 0, v_{n}$ is a weak $H^{1}$ subsolution of $\Delta v+c_{n}(x) v=0$ in the domain $\varepsilon_{n}^{-1} \Lambda$, where $c_{n}(x)=K\left(\varepsilon_{n} x\right) v_{n}^{p-1}(x)+Q\left(\varepsilon_{n} x\right) v_{n}^{q-1}(x)$ and $c_{n}(x) \in L^{s}\left(\varepsilon_{n}^{-1} \Lambda\right)$ with $s \in(N / 2,2 N /((p-1)(N-2)))$. Also, $\left\|c_{n}(x)\right\|_{L^{s}\left(\varepsilon_{n}^{-1} \Lambda\right)}$ is uniformly bounded with respect to $n$, as shown the proof of Lemma 3.4.

By [Gilbarg and Trudinger 1983, Theorem 8.17 and page 193], there is a positive constant $C$ depending only on the dimension $N$ and the $L^{s}\left(\varepsilon_{n}^{-1} \Lambda\right)$ bound of $c_{n}(x)$, such that

$$
v_{n}^{2}\left(\varepsilon_{n}^{-1} x_{\varepsilon_{n}}\right) \leq C \int_{B_{1}\left(\varepsilon_{n}^{-1} x_{\varepsilon_{n}}\right)} v_{n}^{2}(y) d y \leq C \int_{B_{1}\left(\varepsilon_{n}^{-1} x_{\varepsilon_{n}}\right)}\left(\left|\nabla v_{n}\right|^{2}+v_{n}^{2}\right) d y .
$$

Note that $v_{n}\left(\varepsilon_{n}^{-1} x_{\varepsilon_{n}}\right)=u_{\varepsilon_{n}}\left(x_{\varepsilon_{n}}\right)=\max u_{\varepsilon_{n}}$. Then by Lemma 5.1 and (5-7), we get (5-6), which proves (5-4).

By Lemma 2.6, (4-2) and Lemma 4.3, the set $\left\{\mu_{n}\right\}$ satisfies (4-38) up to a subsequence. Then by the argument of Lemmas 4.1 and 4.2 , the set $\left\{\mu_{n}\right\}$ is compact. However, by (5-3), (5-4) and the method that proved Proposition 3.1, we conclude that $\left\{\mu_{n}\right\}$ cannot be compact. This contradiction proves (5-1).

On the other hand, we can prove (5-2) by arguing as in the proof of (3-2).

Lemma 5.4. For any $v>0$, there exist $R(v)>0$ and $\varepsilon_{0}(v)>0$ such that $u_{\varepsilon}(x) \leq v$ for $\varepsilon \leq \varepsilon_{0}(v)$ and $\left|x-x_{\varepsilon}\right| \geq \varepsilon R(v)$. 
Proof. By (2-8), we know that $w_{\varepsilon}(x)=u_{\varepsilon}\left(\varepsilon x+x_{\varepsilon}\right)$ is a classical solution of

$$
\begin{aligned}
-\Delta w_{\varepsilon}+V\left(\varepsilon x+x_{\varepsilon}\right) w_{\varepsilon}=\chi_{\varepsilon}(x) K\left(\varepsilon x+x_{\varepsilon}\right) & w_{\varepsilon}^{p}+\chi_{\varepsilon}(x) Q\left(\varepsilon x+x_{\varepsilon}\right) w_{\varepsilon}^{q} \\
& +\left(1-\chi_{\varepsilon}(x)\right) f_{\varepsilon}\left(\varepsilon x+x_{\varepsilon}, w_{\varepsilon}\right),
\end{aligned}
$$

where $\chi_{\varepsilon}$ is the characteristic function of $A^{\varepsilon}=\left\{\left(x-x_{\varepsilon}\right) / \varepsilon: x \in \Lambda\right\}$.

Let

$$
\begin{aligned}
c_{\varepsilon}(x)=\chi_{\varepsilon}(x) K\left(\varepsilon x+x_{\varepsilon}\right) w_{\varepsilon}^{p-1}(x)+\chi_{\varepsilon}(x) Q\left(\varepsilon x+x_{\varepsilon}\right) & w_{\varepsilon}^{q-1}(x) \\
& +\left(1-\chi_{\varepsilon}(x)\right) \frac{2 \varepsilon^{3}}{1+\left|\varepsilon x+x_{\varepsilon}\right|^{\theta_{0}}} .
\end{aligned}
$$

Then $w_{\varepsilon} \in H^{1}\left(\mathbb{R}^{N}\right)$ is a nonnegative weak subsolution of $\Delta w+c_{\varepsilon}(x) w=0$. Choosing $s \in(N / 2,2 N /((p-1)(N-2)))$ and using the argument that proved Lemma 3.4, we have $c_{\varepsilon}(x) \in L^{s}\left(\mathbb{R}^{N}\right)$ and $\left\|c_{\varepsilon}(x)\right\|_{L^{s}}$ is uniformly bounded with respect to small $\varepsilon$.

Choose a fixed constant $d>0$. Then $B_{d / 2}(x) \subset \mathbb{R}^{N} \backslash B_{\rho(v)}(0)$ holds for any $v>0$ and $x \in \mathbb{R}^{N} \backslash B_{\rho(v)+d}(0)$, where $\rho(v)$ is the constant given in Lemma 5.3. Let $\eta(x)$ be a smooth cutoff function such that $\eta(x)=0$ in $B_{\rho(v)}(0)$ and $\eta(x)=1$ in $\mathbb{R}^{N} \backslash B_{\rho(v)+d / 2}(0)$, with $0 \leq \eta(x) \leq 1$ and $|\nabla \eta| \leq 4 / d$. By [Gilbarg and Trudinger 1983, Theorem 8.17 and page 193], the Sobolev embedding theorem, (2-5) and (5-1), there is a positive constant $C$ depending only on $d$, the dimension $N$ and the $L^{s}$ bound of $c_{\varepsilon}$ such that for small $\varepsilon$ and $x \in \mathbb{R}^{N} \backslash B_{\rho(v)+d}$,

$$
\begin{aligned}
w_{\varepsilon}(x) & \leq C\left(\int_{B_{d / 2}(x)} w_{\varepsilon}^{2^{*}}(y) d y\right)^{1 / 2^{*}} \leq C\left(\int_{\mathbb{R}^{N}}\left(\eta w_{\varepsilon}\right)^{2^{*}}(y) d y\right)^{1 / 2^{*}} \\
& \leq C\left(\int_{\mathbb{R}^{N}}\left|\nabla\left(\eta w_{\varepsilon}\right)\right|^{2}(y) d y\right)^{1 / 2} \\
& \leq C\left(\int_{\mathbb{R}^{N}} \eta^{2}(y)\left|\nabla w_{\varepsilon}\right|^{2}(y)+|\nabla \eta|^{2}(y) w_{\varepsilon}^{2}(y) d y\right)^{1 / 2} \\
& \leq C\left(\int_{\mathbb{R}^{N} \backslash B_{\rho(v)}(0)}\left|\nabla w_{\varepsilon}\right|^{2}(y)+\int_{B_{\rho(v)+d / 2}(0) \backslash B_{\rho(v)}(0)} \frac{16}{d^{2}} w_{\varepsilon}^{2}(y) d y\right)^{1 / 2} \\
& \leq C\left(\int_{\mathbb{R}^{N} \backslash B_{\rho(v)}(0)}\left|\nabla w_{\varepsilon}\right|^{2}(y)+\int_{B_{\rho(v)+d / 2}(0) \backslash B_{\rho(v)}(0)} V\left(\varepsilon x+x_{\varepsilon}\right) w_{\varepsilon}^{2}(y) d y\right)^{1 / 2} \\
& \leq C\left(\int_{\mathbb{R}^{N} \backslash B_{\rho(v)}(0)}\left|\nabla w_{\varepsilon}\right|^{2}(y)+\int_{\mathbb{R}^{N} \backslash B_{\rho(v)}(0)} V\left(\varepsilon x+x_{\varepsilon}\right) w_{\varepsilon}^{2}(y) d y\right)^{1 / 2} \\
& =C\left(\varepsilon^{-N} \int_{\mathbb{R}^{N} \backslash B_{\varepsilon \rho(v)}\left(x_{\varepsilon}\right)}\left(\varepsilon^{2}\left|\nabla u_{\varepsilon}\right|^{2}+V(x) u_{\varepsilon}^{2}\right) d x\right)^{1 / 2} \leq C v^{1 / 2} .
\end{aligned}
$$

Set $R(v)=\rho(v)+d$. Then we get $w_{\varepsilon}(x) \leq v$ for $|x| \geq R(v)$ and small $\varepsilon$. Noting $u_{\varepsilon}(x)=w_{\varepsilon}\left(\left(x-x_{\varepsilon}\right) / \varepsilon\right)$ then finishes the proof. 
Theorem 5.5. For each sequence $\varepsilon_{n}^{\prime}$ such that $\varepsilon_{n}^{\prime} \rightarrow 0$ as $n \rightarrow \infty$, there exists a subsequence $\left\{\varepsilon_{n}\right\} \subset\left\{\varepsilon_{n}^{\prime}\right\}$ such that $u_{n}(x) \equiv u_{\varepsilon_{n}}(x)$ concentrates at some minimum point $x_{0}$ of $G(x)$ in $\Lambda$ as $\varepsilon_{n} \rightarrow 0$, that is, there exists a positive constant $C>0$ such that for any $\delta>0$ and large $n$,

$$
1 / C \leq \max _{\left|x-x_{0}\right| \leq \delta} u_{n} \leq C
$$

and

$$
u_{n}(x) \rightarrow 0 \text { as } n \rightarrow+\infty \text { uniformly with respect to } x \text { for }\left|x-x_{0}\right| \geq \delta .
$$

In particular, if $M=\left\{x \in \Lambda: G(x)=c_{0}\right\}$ consists of only one point $x_{0}$ in $\Lambda$, then all bound states $u_{\varepsilon}$ concentrate at the point $x_{0}$ as $\varepsilon \rightarrow 0$.

Proof. By (5-2), for each sequence $\left\{\varepsilon_{n}^{\prime}\right\}$, there exists a subsequence $\left\{\varepsilon_{n}\right\}$ such that $\left\{x_{n}\right\} \equiv\left\{x_{\varepsilon_{n}}\right\}$ converges to a minimum point $x_{0}$ of $G(x)$ in $\Lambda$ as $n \rightarrow+\infty$, where $x_{n}$ satisfies $u_{n}\left(x_{n}\right)=\max u_{n}(x)$. Given $\delta>0$, we can choose $n$ large enough that $\left|\frac{x-x_{n}}{\varepsilon_{n}}\right|=\left|\frac{x-x_{0}+x_{0}-x_{n}}{\varepsilon_{n}}\right| \geq\left|\frac{x-x_{0}}{\varepsilon_{n}}\right|-\left|\frac{x_{0}-x_{n}}{\varepsilon_{n}}\right|>\frac{\delta}{\varepsilon_{n}}-\frac{\delta}{2 \varepsilon_{n}}=\frac{\delta}{2 \varepsilon_{n}}>R(v)$ for any $v>0$ and $\left|x-x_{0}\right| \geq \delta$, where $R(v)$ is the constant given in Lemma 5.4. This, together with Lemma 5.4, yields $u_{\varepsilon}(x) \leq v$ and thus (5-10).

By Lemma 5.1 and (5-10), we deduce $\max _{\mathbb{R}^{N}} u_{n}=\max _{\left|x-x_{0}\right| \leq \delta} u_{n}$, and the first inequality of (5-9) holds. We now show the second. In fact, by the procedure leading to (5-7) and the last inequality of Lemma 2.6, we have

$$
\begin{aligned}
\max _{\mathbb{R}^{N}} u_{\varepsilon} & =v_{\varepsilon}\left(\varepsilon^{-1} x_{\varepsilon}\right) \leq C\left(\int_{B_{1}\left(\varepsilon^{-1} x_{\varepsilon}\right)} v_{\varepsilon}^{2}(y) d y\right)^{1 / 2} \leq C\left(\int_{B_{1}\left(\varepsilon^{-1} x_{\varepsilon}\right)}\left(\left|\nabla v_{\varepsilon}\right|^{2}+v_{\varepsilon}^{2}\right) d y\right)^{1 / 2} \\
& =C\left(\varepsilon^{-N} \int_{B_{\varepsilon}\left(x_{\varepsilon}\right)}\left(\varepsilon^{2}\left|\nabla u_{\varepsilon}\right|^{2}+\left|u_{\varepsilon}\right|^{2}\right) d x\right)^{1 / 2} \\
& \leq C\left(\varepsilon^{-N} \int_{\Lambda}\left(\varepsilon^{2}\left|\nabla u_{\varepsilon}\right|^{2}+V(x)\left|u_{\varepsilon}\right|^{2}\right) d x\right)^{1 / 2} \leq C .
\end{aligned}
$$

Thus Theorem 5.5 is proved.

Proof of Theorem 1.5. This is an immediate corollary of Theorem 5.5.

\section{Appendix}

Here we prove (2-7).

Lemma A.1. Let

$$
\begin{aligned}
& h_{\varepsilon}(x, \xi)=\min \left\{K(x)\left(\xi^{+}\right)^{p}+2 Q^{+}(x)\left(\xi^{+}\right)^{q}, \frac{\varepsilon^{3}}{1+|x|^{\theta_{0}}} \xi^{+}, \frac{\varepsilon}{1+|x|^{N}}\right\}, \\
& j_{\varepsilon}(x, \xi)=\min \left\{|Q(x)|\left(\xi^{+}\right)^{q}, \frac{\varepsilon^{3}}{1+|x|^{\theta_{0}}} \xi^{+}, \frac{\varepsilon}{1+|x|^{N}}\right\} .
\end{aligned}
$$


Then

$$
\begin{gathered}
\left|h_{\varepsilon}(x, \xi)-h_{\varepsilon}(x, \eta)\right| \leq \frac{p \varepsilon^{3}}{1+|x|^{\theta_{0}}}|\xi-\eta| \quad \text { for } \xi, \eta \in \mathbb{R}, \\
\left|j_{\varepsilon}(x, \xi)-j_{\varepsilon}(x, \eta)\right| \leq \frac{q \varepsilon^{3}}{1+|x|^{\theta_{0}}}|\xi-\eta| \quad \text { for } \xi, \eta \in \mathbb{R} .
\end{gathered}
$$

Proof. We only prove (A-1). Because $\left|\xi^{+}-\eta^{+}\right| \leq|\xi-\eta|$, it suffices to show (A-1) for $\xi, \eta \geq 0$. We note that (A-1) obviously holds for $\xi=\eta$, and $h_{\varepsilon}(x, \xi)$ is not decreasing for $\xi \geq 0$. So we can assume $\xi>\eta \geq 0$ without loss of generality. We now treat various cases and subcases.

Case I: $\eta=0$. In this case,

$$
0 \leq h_{\varepsilon}(x, \xi)-h_{\varepsilon}(x, \eta)=h_{\varepsilon}(x, \xi) \leq \frac{\varepsilon^{3}}{1+|x|^{\theta_{0}}} \xi<\frac{p \varepsilon^{3}}{1+|x|^{\theta_{0}}}(\xi-\eta) .
$$

Case II: $\eta>0$.

Case II.1: $h_{\varepsilon}(x, \xi)=K(x) \xi^{p}+2 Q^{+}(x) \xi^{q}$. Then, because $\xi>\eta$, we have $h_{\varepsilon}(x, \eta)=K(x) \eta^{p}+2 Q^{+}(x) \eta^{q}$. It follows from the definition of $h_{\varepsilon}(x, \xi)$ and a direct computation that $h_{\varepsilon}(x, \xi)-h_{\varepsilon}(x, \eta)<p \varepsilon^{3}(\xi-\eta) /\left(1+|x|^{\theta_{0}}\right)$.

Case II.2: $h_{\varepsilon}(x, \xi)=\varepsilon^{3} \xi /\left(1+|x|^{\theta_{0}}\right)$. By $\xi>\eta$, we have

$$
h_{\varepsilon}(x, \eta)=K(x) \eta^{p}+2 Q^{+}(x) \eta^{q} \quad \text { or } \quad h_{\varepsilon}(x, \eta)=\varepsilon^{3} \eta /\left(1+|x|^{\theta_{0}}\right) .
$$

Case II.2.i: $h_{\varepsilon}(x, \eta)=K(x) \eta^{p}+2 Q^{+}(x) \eta^{q}$. Denote by $w$ the unique positive solution of $\varepsilon^{3} /\left(1+|x|^{\theta_{0}}\right)=K(x) w^{p-1}+2 Q^{+}(x) w^{q-1}$; at this time, $K(x) \neq 0$ or $Q^{+}(x) \neq 0$ by the definition of $h_{\varepsilon}(x, \xi)$. Then it follows from $\eta \leq w \leq \xi$ that $h_{\varepsilon}(x, w)=K(x) w^{p}+2 Q^{+}(x) w^{q}=\varepsilon^{3} w /\left(1+|x|^{\theta_{0}}\right)$. Thus

$$
\begin{aligned}
& h_{\varepsilon}(x, \xi)-h_{\varepsilon}(x, \eta)=h_{\varepsilon}(x, \xi)-h_{\varepsilon}(x, w)+h_{\varepsilon}(x, w)-h_{\varepsilon}(x, \eta) \\
&= \frac{\varepsilon^{3}}{1+|x|^{\theta_{0}}}(\xi-w)+K(x)\left(w^{p}-\eta^{p}\right)+2 Q^{+}(x)\left(w^{q}-\eta^{q}\right) \\
&= \frac{\varepsilon^{3}}{1+|x|^{\theta_{0}}}(\xi-w)+p K(x) \zeta_{1}{ }^{p-1}(w-\eta)+2 q Q^{+}(x) \zeta_{2}{ }^{q-1}(w-\eta) \\
& \quad \quad\left(\text { where } \eta \leq \zeta_{1}, \zeta_{2} \leq w\right) \\
& \quad \leq \frac{\varepsilon^{3}}{1+|x|^{\theta_{0}}}(\xi-w)+p\left(K(x) w^{p-1}+2 Q^{+}(x) w^{q-1}\right)(w-\eta) \\
&=\frac{\varepsilon^{3}}{1+|x|^{\theta_{0}}}(\xi-w)+\frac{p \varepsilon^{3}}{1+|x|^{\theta_{0}}}(w-\eta) \leq \frac{p \varepsilon^{3}}{1+|x|^{\theta_{0}}}(\xi-\eta) .
\end{aligned}
$$

Case II.2.ii: $h_{\varepsilon}(x, \eta)=\varepsilon^{3} \eta /\left(1+|x|^{\theta_{0}}\right)$. It follows from a direct computation that

$$
h_{\varepsilon}(x, \xi)-h_{\varepsilon}(x, \eta)=\varepsilon^{3}(\xi-\eta) /\left(1+|x|^{\theta_{0}}\right)<p \varepsilon^{3}(\xi-\eta) /\left(1+|x|^{\theta_{0}}\right) .
$$


Case II.3: $h_{\varepsilon}(x, \xi)=\varepsilon /\left(1+|x|^{N}\right)$. In this case, $h_{\varepsilon}(x, \eta)$ is either

$$
K(x) \eta^{p}+2 Q^{+}(x) \eta^{q} \quad \text { or } \quad \varepsilon^{3} \eta /\left(1+|x|^{\theta_{0}}\right) \quad \text { or } \quad \varepsilon /\left(1+|x|^{N}\right) .
$$

Case II.3.i: $h_{\varepsilon}(x, \eta)=K(x) \eta^{p}+2 Q^{+}(x) \eta^{q}$. If $\xi \geq w$, with $w$ as in Case II.2.i, then we have

$$
\begin{aligned}
h_{\varepsilon}(x, \xi)-h_{\varepsilon}(x, \eta)=\frac{\varepsilon}{1+|x|^{N}}-\left(K(x) \eta^{p}+2 Q^{+}(x) \eta^{q}\right) \\
\quad \leq \frac{\varepsilon^{3}}{1+|x|^{\theta_{0}}} \xi-\left(K(x) \eta^{p}+2 Q^{+}(x) \eta^{q}\right) \\
\quad=\frac{\varepsilon^{3}}{1+|x|^{\theta_{0}}}(\xi-w)+K(x)\left(w^{p}-\eta^{p}\right)+2 Q^{+}(x)\left(w^{q}-\eta^{q}\right) \\
\quad=\frac{\varepsilon^{3}}{1+|x|^{\theta_{0}}}(\xi-w)+p K(x) \zeta_{1}^{p-1}(w-\eta)+2 q Q^{+}(x) \zeta^{q-1}(w-\eta) \\
\quad \leq \frac{\varepsilon^{3}}{1+|x|^{\theta_{0}}}(\xi-w)+p\left[K(x) w^{p-1}+2 Q^{+}(x) w^{q-1}\right](w-\eta) \\
\quad=\frac{\varepsilon^{3}}{1+|x|^{\theta_{0}}}(\xi-w)+\frac{p \varepsilon^{3}}{1+|x|^{\theta_{0}}}(w-\eta) \leq \frac{p \varepsilon^{3}}{1+|x|^{\theta_{0}}}(\xi-\eta) .
\end{aligned}
$$

If $\xi<w$, then $\varepsilon /\left(1+|x|^{N}\right) \leq K(x) \xi^{p}+2 Q^{+}(x) \xi^{q} \leq \varepsilon^{3} /\left(1+|x|^{\theta_{0}}\right) \xi$. A direct computation yields

$$
\begin{aligned}
h_{\varepsilon}(x, \xi) & -h_{\varepsilon}(x, \eta)=\frac{\varepsilon}{1+|x|^{N}}-\left(K(x) \eta^{p}+2 Q^{+}(x) \eta^{q}\right) \\
& \leq\left(K(x) \xi^{p}+2 Q^{+}(x) \xi^{q}\right)-\left(K(x) \eta^{p}+2 Q^{+}(x) \eta^{q}\right) \\
& =K(x)\left(\xi^{p}-\eta^{p}\right)+2 Q^{+}(x)\left(\xi^{q}-\eta^{q}\right) \\
& =p K(x) \zeta_{1}^{p-1}(\xi-\eta)+2 q Q^{+}(x) \zeta_{2}^{q-1}(\xi-\eta) \quad\left(\text { where } \eta \leq \zeta_{1}, \zeta_{2} \leq \xi\right) \\
& \leq p\left(K(x) \xi^{p-1}+2 Q^{+}(x) \xi^{q-1}\right)(\xi-\eta) \\
& \leq \frac{p \varepsilon^{3}}{1+|x|^{\theta_{0}}}(\xi-\eta) .
\end{aligned}
$$

Case II.3.ii: $h_{\varepsilon}(x, \eta)=\varepsilon^{3} \eta /\left(1+|x|^{\theta_{0}}\right)$. It follows from the definition of $h_{\varepsilon}(x, \eta)$ and a direct computation that

$h_{\varepsilon}(x, \xi)-h_{\varepsilon}(x, \eta)=\frac{\varepsilon}{1+|x|^{N}}-\frac{\varepsilon^{3}}{1+|x|^{\theta_{0}}} \eta \leq \frac{\varepsilon^{3}}{1+|x|^{\theta_{0}}}(\xi-\eta)<\frac{p \varepsilon^{3}}{1+|x|^{\theta_{0}}}(\xi-\eta)$.

Case II.3.iii: $h_{\varepsilon}(x, \eta)=\varepsilon /\left(1+|x|^{N}\right)$. We have

$$
h_{\varepsilon}(x, \xi)-h_{\varepsilon}(x, \eta)=0<p \varepsilon^{3}(\xi-\eta) /\left(1+|x|^{\theta_{0}}\right) .
$$

Combining all the cases above yields (A-1). 


\section{References}

[Ambrosetti and Malchiodi 2007] A. Ambrosetti and A. Malchiodi, "Concentration phenomena for nonlinear Schrödinger equations: recent results and new perspectives", pp. 19-30 in Perspectives in nonlinear partial differential equations, edited by H. Berestycki et al., Contemp. Math. 446, Amer. Math. Soc., Providence, RI, 2007. MR 2008j:35037

[Ambrosetti et al. 2003] A. Ambrosetti, A. Malchiodi, and W.-M. Ni, "Singularly perturbed elliptic equations with symmetry: existence of solutions concentrating on spheres, I", Comm. Math. Phys. 235:3 (2003), 427-466. MR 2004c:35014 Zbl 1072.35019

[Ambrosetti et al. 2004] A. Ambrosetti, A. Malchiodi, and W.-M. Ni, "Singularly perturbed elliptic equations with symmetry: existence of solutions concentrating on spheres, II", Indiana Univ. Math. J. 53:2 (2004), 297-329. MR 2005c:35015 Zbl 1081.35008

[Ambrosetti et al. 2005] A. Ambrosetti, V. Felli, and A. Malchiodi, "Ground states of nonlinear Schrödinger equations with potentials vanishing at infinity”, J. Eur. Math. Soc. 7:1 (2005), 117144. MR 2006f:35049

[Ambrosetti et al. 2006] A. Ambrosetti, A. Malchiodi, and D. Ruiz, "Bound states of nonlinear Schrödinger equations with potentials vanishing at infinity", J. Anal. Math. 98 (2006), 317-348. MR 2007f:35071

[Bonheure and Van Schaftingen 2008] D. Bonheure and J. Van Schaftingen, "Bound state solutions for a class of nonlinear Schrödinger equations", Rev. Mat. Iberoam. 24:1 (2008), 297-351. MR 2009d:35069

[Byeon and Wang 2003] J. Byeon and Z.-Q. Wang, "Standing waves with a critical frequency for nonlinear Schrödinger equations, II", Calc. Var. Partial Differential Equations 18:2 (2003), 207219. MR 2004h:35207

[Cao and Peng 2006] D. Cao and S. Peng, "Multi-bump bound states of Schrödinger equations with a critical frequency”, Math. Ann. 336:4 (2006), 925-948. MR 2008a:35259

[Cingolani 2003] S. Cingolani, "Semiclassical stationary states of nonlinear Schrödinger equations with an external magnetic field", J. Differential Eq. 188:1 (2003), 52-79. MR 2004a:81096

[Cingolani and Lazzo 2000] S. Cingolani and M. Lazzo, "Multiple positive solutions to nonlinear Schrödinger equations with competing potential functions", J. Differential Eq. 160:1 (2000), 118138. MR 2000j:35079

[Ding and Tanaka 2003] Y. Ding and K. Tanaka, "Multiplicity of positive solutions of a nonlinear Schrödinger equation”, Manuscripta Math. 112:1 (2003), 109-135. MR 2004i:35099

[Floer and Weinstein 1986] A. Floer and A. Weinstein, "Nonspreading wave packets for the cubic Schrödinger equation with a bounded potential”, J. Funct. Anal. 69:3 (1986), 397-408. MR 88d: 35169

[Gidas et al. 1981] B. Gidas, W. M. Ni, and L. Nirenberg, "Symmetry of positive solutions of nonlinear elliptic equations in $\mathbb{R}^{n}$ ", pp. 369-402 in Mathematical analysis and applications, Part A, edited by L. Nachbin, Adv. in Math. Suppl. Stud. 7, Academic Press, New York, 1981. MR 84a:35083 Zbl 0469.35052

[Gilbarg and Trudinger 1983] D. Gilbarg and N. S. Trudinger, Elliptic partial differential equations of second order, 2nd ed., Grundlehren der Mathematischen Wissenschaften 224, Springer, Berlin, 1983. MR 86c:35035 Zbl 0562.35001

[Grossi 2002] M. Grossi, "On the number of single-peak solutions of the nonlinear Schrödinger equation”, Ann. Inst. H. Poincaré Anal. Non Linéaire 19:3 (2002), 261-280. MR 2003k:35228

[Gui 1996] C. Gui, "Existence of multi-bump solutions for nonlinear Schrödinger equations via variational method”, Comm. Partial Differential Equations 21:5-6 (1996), 787-820. MR 98a:35122 


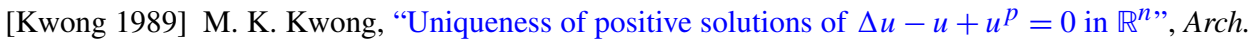
Rational Mech. Anal. 105:3 (1989), 243-266. MR 90d:35015 Zbl 0676.35032

[Lions 1984a] P.-L. Lions, "The concentration-compactness principle in the calculus of variations: The locally compact case, I”, Ann. Inst. H. Poincaré Anal. Non Linéaire 1:2 (1984), 109-145. MR 87e:49035a Zbl 0541.49009

[Lions 1984b] P.-L. Lions, "The concentration-compactness principle in the calculus of variations: The locally compact case, II”, Ann. Inst. H. Poincaré Anal. Non Linéaire 1:4 (1984), 223-283. MR 87e:49035b

[Ni 1982] W. M. Ni, "On the elliptic equation $\Delta u+K(x) u^{(n+2) /(n-2)}=0$, its generalizations, and applications in geometry", Indiana Univ. Math. J. 31:4 (1982), 493-529. MR 84e:35049 Zbl 0496.35036

[Oh 1990] Y.-G. Oh, "On positive multi-lump bound states of nonlinear Schrödinger equations under multiple well potential”, Comm. Math. Phys. 131:2 (1990), 223-253. MR 92a:35148

[del Pino and Felmer 1996] M. del Pino and P. L. Felmer, "Local mountain passes for semilinear elliptic problems in unbounded domains", Calc. Var. Partial Differential Equations 4:2 (1996), 121-137. MR 97c:35057 Zbl 0844.35032

[Rabinowitz 1992] P. H. Rabinowitz, "On a class of nonlinear Schrödinger equations", Z. Angew. Math. Phys. 43:2 (1992), 270-291. MR 93h:35194

[Wang 1993] X. Wang, "On concentration of positive bound states of nonlinear Schrödinger equations”, Comm. Math. Phys. 153:2 (1993), 229-244. MR 94m:35287

[Wang and Zeng 1997] X. Wang and B. Zeng, "On concentration of positive bound states of nonlinear Schrödinger equations with competing potential functions”, SIAM J. Math. Anal. 28:3 (1997), 633-655. MR 98e:81032

[Yin and Zhang 2009] H. Yin and P. Zhang, "Bound states of nonlinear Schrödinger equations with potentials tending to zero at infinity”, J. Differential Eq. 247:2 (2009), 618-647. MR 2523695

Received March 1, 2009. Revised September 2, 2009.

\author{
MingWen FeI \\ DEPARTMENT OF MATHEMATICS AND IMS \\ NANJING UNIVERSITY \\ NANJING 210093 \\ CHINA \\ ahnufmwen@126.com \\ HUICHENG YIN \\ DEPARTMENT OF MATHEMATICS AND IMS \\ NANJING UNIVERSITY \\ NANJING 210093 \\ CHINA
}

huicheng@nju.edu.cn 